\title{
Assessing the potential impact of hormonal based contraceptives on HIV transmission dynamics among heterosexuals
}

\author{
Noble. J. Malunguza ${ }^{1 *}$ Senelani D. Hove-Musekwa ${ }^{1}$, Zindoga Mukandavire ${ }^{2}$, \\ ${ }^{1}$ Department of Applied Mathematics, National University of Science and Technology, Bulawayo, Zimbabwe \\ ${ }^{2}$ Social and Mathematical Epidemiology Group, London School of Hygiene and Tropical Medicine, London, UK
}

\begin{abstract}
Background: HIV susceptibility linked to hormonal contraception (HC) has been studied before, but with mixed results. Recent findings have prompted the World Health Organisation (WHO), to encourage women who use HC to concurrently use condoms in order to prevent HIV infection in light of possible increased HIV risk of infection associated with hormone based contraceptives.

Methods and Findings: A two sex HIV model classifying women into three risk groups consisting of individuals who use condoms, natural methods and hormone based contraceptives is formulated and analysed to assess the effects of various birth control methods on the transmission dynamics of the disease. Our model results showed that women who use HC could be key drivers of the epidemic and that their increased infectivity may be critical in driving the epidemic. Women who use hormone based contraceptives potentially act as a core-group from which men get infected and in turn transmit the disease to other population groups. We fitted the model to HIV prevalence data for Zimbabwe reported by UNAIDS and Zimbabwe Ministry of Health and Child welfare (MoHCW) and used the model fit to project HIV prevalence. Predictions using HIV data for Zimbabwe suggest that a hypothesised increase in susceptibility and infectivity of 2,3 , and 4 fold would result in a $25 \%, 50 \%$ and $100 \%$ increase in baseline HIV prevalence projection respectively, thus suggesting possible increased disease burden even in countries reporting plausible HIV prevalence declines.

Conclusion:Although a possible causal relationship between HIV susceptibility and HC use remains subject of continuing scientific probe, its inclusion as part of birth control strategy, has been shown in this study, to possibly have significant influence on HIV transmission. If proven, HC use may potentially explain the inordinate spread of HIV within the sub-Saharan Africa region and therefore compel for urgent assessment with a view to reorienting birth control methods in use in settings with generalised epidemics.
\end{abstract}

Key words: Hormonal contraceptives, heightened HIV transmissions, reproductive number, stability, model fitting.

\section{Introduction}

Despite declining birth and increasing death rates due to AIDS, sub-Saharan Africa's population has doubled to 620 million, in less than 25 years and is projected to double again within thirty years [72]. A tenth of the world's female population lives in sub-Saharan Africa, but the region accounts for $40 \%$ of the world's 215, 000 annual pregnancy-related deaths [72]. Sub-Saharan Africa is home to only $11 \%$ of the world's population, yet by the close of 2001, it was habitat to $70 \%$ of the world's 40 million people living with HIV/AIDS [43]. Unfortunately there is no evidence that blending of AIDS education into

${ }^{*}$ Correspondence: Noble. J. Malunguza, Email: nmalunguza@gmail.com,nmalunguza@nust.ac.zw 
family planning and maternal health services is taking place. Studies in 10 sub-Saharan countries [72], showed that health staff discuss sexually transmitted diseases with only 1 in 10 family planning clients and AIDS with just 1 in 14 and yet such services often are the only contact women have with the health system. An important opportunity for educating women about protecting themselves from HIV and AIDS is thus missed. In sub-Saharan Africa, $23 \%$ of married women are using family planning, $18 \%$ with a modern method, $5 \%$ with a traditional method [24] and $25 \%$ reporting that they would prefer to stop having children or delay their next birth, but are not using any method of family planning. The average contraceptive prevalence in this region (22\%), is less than half that of South Asia (53\%) and less than a third that of East Asia (77\%) [89].

Diverse birth control strategies are practised in the region. The natural (traditional) method of birth control which is menstrual cycle dependent, is favoured and often prescribed as the only correct one among some religious groups. In Kenya studies showed that the probability of a woman using family planning services if she was Catholic was $28 \%$ lower than that of women with different religious background such as Protestant and Muslim [69]. Yeatman and Trinitapoli [90], however showed that Muslims were the least likely to use injectables, pills, Intra Uterine Devices or condoms. Some churches discourage faithfuls from using contraceptives as birth control measures and instead encourage them to rely more on the natural method [69] and expressly forbid the use of barrier methods of contraception such as condoms while treating emergency contraception such as the morning-after pill as a means of abortion [12]. Nearly $30 \%$ of women in sub-Saharan Africa do not use birth control as they are either opposed to contraception, unaware of options, have problems with access or believed they were not fertile [2] and would prefer birth control methods with no contraceptive effects, were compatible with infrequent sex and whose use would be non-detectable to their sex partner. Barrier methods involve the use of rubber products that prevent the exchange of mucosal fluids during intercourse and the most commonly used are condoms. Hormonal methods of birth control involve the ingestion or the contraceptive injection of a substance whose main ingredient is progesterone administered via a tablet or some liquid. Andreea et al. [14], showed that short term family planning methods involving contraceptives were more popular ( $6 \%$ of women between the ages of 15 and 49 having used injectables) compared with long term methods, with the pill being favoured by teenagers, women who are cohabiting, women with no children and women with at least a college education. Women in the richest wealth quintile were more likely than those in the poorest quintile to practice long-term contraception such as intrauterine devices, implants and sterilisation.

The UN, at its 2000 summit set the improvement of maternal health by 2015 as its $5^{\text {th }}$ MDG [79]. This was to be achieved through provision of universal access to reproductive health. Combating of HIV/AIDS by halting and reversing its spread by 2015 was adopted as the $6^{\text {th }}$ MDG. Evidence that the use of HC methods to attain maternal health for all by 2015 could be impacting negatively on efforts to combat HIV spread in the region that suffers most from unintended pregnancies (59\%) [73] and high HIV prevalence rates in excess of $10 \%[77,78,80-82]$ pose a new dilemma and challenges to global development [25]. The implications are not palatable given that the region is afflicted with high maternal and neonatal mortality rates which make access to contraception a necessity. As early as 2004, HC use was already being cited as a possible risk factor for HIV transmission [88]. According to recent studies [28,70], women who use hormonal methods of birth control such as the pill or the contraceptive injection had a significantly higher risk of HIV-1 seroconversion. Heffron et al. [28] reported two fold increases in the risks of both HIV acquisition and HIV transmission among women in HIV discordant couples. Results of an analogous study in macaques [50] showed that female rhesus macaques with progesterone implants were 7.7 times more likely to be infected with simian immunodeficiency virus than macaques that had a placebo implant. Other studies reporting increased risk of HIV acquisition in women and possible increased risk of female-to-male HIV transmission include [3, 40, 45, 65-67, 70]. 
However results from studies by $[37,38,62]$ reported no association between HIV acquisition risk and use of injectable contraception. The dichotomy in the results is amplified by discrepancies in study design, contraceptive method studied, sample sizes, analytic methods used, and the complexity of interpreting results across these observational studies [26]. In a systematic review [67], four of nine studies considered informative but with important limitations reported a 1.5 to 2.2 times increased risk of HIV acquisition in women using injectable contraception(DMPA) [3, 28, 56, 87]. The remaining five studies of injectable contraception in the review $[39,57,62,71]$ reported no statistically significant association with risk of HIV acquisition. In the same review [67], only one study reported increased risk of acquisition resulting from the use of oral contraceptive pills (OCPs).

Women who took injectable contraceptives had raised concentrations of HIV genetic material in their cervical secretion [28] and this could be the mechanism for passing on the virus to a sexual partner. Injected in the form of tiny crystals suspended in solution, DMPA gradually seeps into the bloodstream, duping a woman's body into thinking she is already pregnant and thus halting ovulation. Thus a woman's defenses against disease may then be lowered in the same manner akin to pregnancy. The thinning of the vaginal mucosa by progesterone has been reported to make it easier for HIV to penetrate the vaginal wall in an uninfected woman, and, conversely, for an HIV-positive woman to infect her partner [86]. Other possible suggestions were that Depo-Provera could result in the reduction of the production of hydrogen peroxide producing lactobacilli which in turn could decrease vaginal acidity as well as cause irregular uterine bleeding $[4,52]$. Progesterone and oestrogen may accentuate recruitment of inflammatory and other target cells to the genital tract [54] although this is considered a very new area of research [27].

In view of this increasing conflicting literature on the link between $\mathrm{HC}$ use and heightened HIV transmission and acquisition risk, WHO experts convened a meeting to review existing literature on the subject [84]. The UN body ruled that available data was insufficient and did not establish a clear causal link between heightened HIV acquisition and transmission and the use of HCs. However they also conceded that available evidence could not conclusively rule out the possibility of an association. Hence its current recommendation for women to continue using HCs to avoid unplanned pregnancies while encouraging women at risk of or living with HIV to use condoms concurrently to prevent HIV infection or transmission. While debate on the HIV link to Depo-Provera rages, we are compelled to develop a novel theoretical HIV model that incorporates, the dynamics of reproductive health in sub-Saharan Africa and includes possible accentuated HIV risks due to $\mathrm{HC}$ use, in order to understand how these affect disease transmission dynamics. HIV models that combine biological and social aspects of populations in subSaharan Africa, such as birth control, alcohol consumption [47,75] and gender inequality [61] are poised to improve our understanding of the disease. To the best of our knowledge, no mathematical modelling studies of the possible dynamic influence of HC use on HIV transmission have been carried out and ours is pioneering work in assessing the potential dynamic impact on HIV transmission of HC use in high HIV prevalence settings.

\section{Model development}

We develop a deterministic compartmental model that classifies the heterosexually active male population into three classes: susceptibles $S_{m}(t)$, containing individual males who are not infected with the virus, $I_{m}(t)$, containing individual males who are infected with the virus but have yet to develop AIDS symptoms and $A_{m}(t)$, are individual male AIDS cases exhibiting specific clinical features related to the disease. Susceptible females, and infectives who have sero-converted but are yet to progress to AIDS are categorised according to the contraceptive method used. Susceptible females and infectives who use condoms for birth control are denoted by $S_{f_{c}}$ and $I_{f_{c}}$ respectively. Conversely, susceptibles and infectives who use the natural method of contraception are denoted by $S_{f_{n}}$ and $I_{f_{n}}$ respectively and those who use 
$\mathrm{HC}$ are denoted by $S_{f_{h}}$ and $I_{f_{h}}$ depending on whether they are infected or not. Females who show clinical features of the disease are categorized in the AIDS class $A_{f}$ and are assumed to be nonreproductive. At any moment in time, new recruits enter the heterosexually active population at a constant rate $\Lambda$. A proportion $\phi$ of susceptible individuals are assumed to be female and the complementary proportion $(1-\phi)$, are male. A proportion $\delta_{1}, \delta_{2}$ and $\delta_{3}$ of new female recruits enter the class of women who use condoms $S_{f_{c}}$, women who use the natural method $S_{f_{n}}$ and women who use HC methods $S_{f_{h}}$ of birth control respectively. It is assumed that a woman will use one method of birth control throughout her reproductive lifetime and that the reproductive lifetime equates to her mean sexual life time. Men can choose any sexual partner irrespective of their contraceptive method. The time dependent force of infection for females who use condoms for birth control is given by $\lambda_{f_{c}}(t)$, and that for females who use the natural method is given by $\lambda_{f_{n}}(t)$. Females that use HC acquire infection at time dependent force of infection $\lambda_{f_{h}}(t)$. Similarly susceptible males acquire infection at time dependent rate $\lambda_{m}(t)$. The infection rate depends on the probability of transmission by an infecting partner $\left(\beta_{j}\right)$, frequency of partner acquisition, $\left(c_{i}\right)$ where $i, j=\left(f_{c}, f_{n}, f_{h}, m\right), i \neq j$ (refers to male or female and excludes same sex interaction) and the proportion of infected individuals in each category. Females who use condoms as a method of birth control confer protection from HIV to their partners and themselves. The protective effect of condom use on HIV transmission is modelled by $1-p_{c}$ where $p_{c}=\epsilon \psi$, with the parameters $\epsilon$ and $\psi$ modeling condom efficacy and consistency respectively. Females who use HC are more susceptible to the virus at a relative measure of susceptibility $\chi>1[28,70]$ and they are at increased risk of passing on the virus to their partner at a relative measure of infectivity $\theta>1$ [28].

Following the approach in $[10,33,34,47]$ on the mixing pattern, we let $q_{i j}$ be the preference of people in group $i$ to have a partner in group $j$, then $q_{i j}$ is the fraction of people in group $j$ that a person in group $i$ desires to form a partnership with. Further details on the formulation of partnership formation and preferences can be found in $[9,11,16,20,30,36,63]$. If we assume an unlimited number of potential partners then the probability that a partnership forms (mutually accepted rate of sex) between people in group $i$ and people in group $j, \rho_{i j}$ is given by $\rho_{i j}=q_{i j} q_{j i}$. Let $c_{i}$ be the number of contacts for an individual in group $i$, and assuming that mixing is random but constrained by the activity levels [63], the probability that a contact in group $i$ is with a person in group $j$ is given by $\frac{c_{j} N_{j}}{\sum_{k} c_{k} N_{k}}$ where $k$ is the number of classes and $N_{k}=S_{k}+I_{k}$ is the total sexually interactive population. The probability of a partnership forming between people in group $i$ and $j$ is then given by $\frac{\rho_{i j} c_{j} N_{j}}{\sum_{k} c_{k} N_{k}}$. The force of infection for susceptible people in group $i$ is thus given by

$$
\lambda_{i}=\sum_{j=1}^{K} \rho_{i j} \beta_{j} \frac{c_{j} I_{j}}{\sum_{k} c_{k} N_{k}} .
$$

where $\beta_{j}$ is the probability of being infected by an individual in group $j$ and $c_{j}$ is the rate of acquiring sexual partners in group $j$. The number of contacts made by people in group $i$ with people in group $j$ must essentially be equal to the number of contacts made by people in group $j$ with people in group $i$ and therefore the $\rho_{i j}$ 's and $c_{i}$ 's must satisfy the following balance equation,

$$
\frac{c_{j} \rho_{j i}}{N_{i}}=\frac{c_{i} \rho_{i j}}{N_{j}} .
$$

Thus the total number of contacts made in unit time by members of group $i$ with members of group $j$ is $c_{i} \rho_{i j} N_{j}$ and because this must equal the total number of contacts by members of group $j$ with members of group $i$, the following balance relations hold

$$
c_{f_{c}} N_{f_{c}} \rho_{f_{c} m}=c_{m} N_{m} \rho_{m f_{c}}, c_{f_{n}} N_{f_{n}} \rho_{f_{n} m}=c_{m} N_{m} \rho_{m f_{n}}, c_{f_{h}} N_{f_{h}} \rho_{f_{h} m}=c_{m} N_{m} \rho_{m f_{h}},
$$


where $N_{f_{c}}, N_{f_{n}}$, and $N_{f_{h}}$ are the respective female populations that use condoms, normal birth control methods and $\mathrm{HC}$ methods and $N_{m}$ is the total sexually interacting male population. Equation (3) implies that the number of contacts with men reported by users of condoms for birth control must equal the number of sexual contacts reported by men with females who use condoms for birth control. Assuming $c_{j}=c_{k}$ that is sexual activity is independent of birth control method used, then the balance law in equation (2) can be written as

$$
\frac{c_{f}}{N_{m}}=\frac{c_{m}}{N_{f_{c}} \rho_{f_{c} m}+N_{f_{n}} \rho_{f_{n} m}+N_{f_{h}} \rho_{f_{h} m}} .
$$

We use birth control as the risk factor and therefore women select their partners without discrimination. Thus $q_{i m}=q_{i}$ and $\rho_{i j}=q_{i} q_{m i}$ and thus $\rho_{f_{c} m}=\rho_{m f_{c}}=q_{f} q_{m f_{c}}, \rho_{f_{n} m}=\rho_{m f_{n}}=q_{f_{n}} q_{m f_{n}}$ and $\rho_{m f_{h}}=$ $q_{f_{h}} q_{m f_{h}}$ where $q_{f_{c}}, q_{f_{n}}$ and $q_{f_{h}}$ is the desire, by females who use condoms, females who use the natural method and females who use HC methods of birth control respectively, to form partnerships with males and $q_{m f_{c}}, q_{m f_{n}}$ and $q_{m f_{h}}$ measures male desires, to form partnerships with females who use condoms, natural methods and HCs respectively. The mixing assumptions lead to the following forces of infection for the different groups.

$$
\left\{\begin{array}{l}
\lambda_{m}(t)=\beta_{f} c_{m} \frac{\rho_{m f_{c}}\left(1-p_{c}\right) I_{f_{c}}(t)+\rho_{m f_{n}} I_{f_{n}}(t)+\rho_{m f_{h}} \theta I_{f_{h}}(t)}{N_{f}(t)}, \\
\lambda_{f_{c}}(t)=\rho_{f_{c} m}\left(1-p_{c}\right) c_{f} \beta_{m} \frac{I_{m}(t)}{N_{m}(t)} \\
\lambda_{f_{n}}(t)=\rho_{f_{n} m} c_{f} \beta_{m} \frac{I_{m}(t)}{N_{m}(t)}, \\
\lambda_{f_{h}}(t)=\rho_{f_{h} m} \chi c_{f} \beta_{m} \frac{I_{m}(t)}{N_{m}(t)}
\end{array}\right.
$$

where $N_{j}$ is the total sexually active population in sex category $j$ (excluding AIDS patients who are assumed to be symptomatic and therefore incapable of new partnership formation). Upon becoming infected with HIV, female and male susceptibles enter the classes $I_{f_{c}}, I_{f_{n}}, I_{f_{h}}$ and $I_{m}$ of infected individuals, respectively. In the context of Zimbabwe whose data we will use for model fitting, we assumed a constant emigration rate $\alpha>0$ of individuals to other countries except for the AIDS patients because the country faced increased emigration due to more than a decade of economic meltdown. Infected individuals in each class progress to AIDS at a constant rate $\gamma>0$. The natural death rate $\mu>0$ is assumed to be proportional to the number in each class. AIDS patients have an additional disease-induced mortality rate, $\nu>0$. In this study we ignore the population which uses long-term methods such as the intrauterine devices, implants and sterilisation, as studies have shown this population to be very small [14] (in sub-Saharan Africa). The model flow diagram is depicted in Figure 1.

The model assumptions and formulations described result in the following deterministic compartmen- 
tal model.

$$
\begin{aligned}
& \frac{d S_{f_{c}}(t)}{d t}=\delta_{1} \phi \Lambda-\lambda_{f_{c}} S_{f_{c}}(t)-(\mu+\alpha) S_{f_{c}}(t), \\
& \frac{d S_{f_{n}}}{d t}(t)=\delta_{2} \phi \Lambda-\lambda_{f_{n}} S_{f_{n}}(t)-(\mu+\alpha) S_{f_{n}}(t), \\
& \frac{d S_{f_{h}}(t)}{d t}=\delta_{3} \phi \Lambda-\lambda_{f_{h}} S_{f_{h}}(t)-(\mu+\alpha) S_{f_{h}}(t), \\
& \frac{d I_{f_{c}}(t)}{d t}=\lambda_{f_{c}} S_{f_{c}}(t)-(\gamma+\mu+\alpha) I_{f_{c}}(t), \\
& \frac{d I_{f_{n}}(t)}{d t}=\lambda_{f_{n}} S_{f_{n}}(t)-(\gamma+\mu+\alpha) I_{f_{n}}(t), \\
& \frac{d I_{f_{h}}(t)}{d t}=\lambda_{f_{h}} S_{f_{h}}(t)-(\gamma+\mu+\alpha) I_{f_{h}}(t), \\
& \frac{d A_{f}(t)}{d t}=\gamma\left(I_{f_{c}}(t)+I_{f_{n}}(t)+I_{f_{h}}(t)\right)-(\mu+\nu) A_{f}(t), \\
& \frac{d S_{m}(t)}{d t}=(1-\phi) \Lambda-\lambda_{m} S_{m}(t)-(\mu+\alpha) S_{m}(t), \\
& \frac{d I_{m}(t)}{d t}=\lambda_{m} S_{m}(t)-(\gamma+\mu+\alpha) I_{m}(t), \\
& \frac{d A_{m}(t)}{d t}=\gamma I_{m}(t)-(\mu+\nu) A_{m}(t) .
\end{aligned}
$$

In the following sections, we will mix analytical and numerical techniques to present a detailed analysis of the model which is followed by fitting of the model to HIV prevalence for Zimbabwe using data reported by UNAIDS [76] and MoHCW [91].

\section{Model basics}

Theorem 1. Given $S_{f_{c}}(0)>0, S_{f_{n}}(0)>0, S_{f_{h}}>0, I_{f_{c}}(0)>0, I_{f_{n}}(0)>0, I_{f_{h}}>0, A_{f}(0)>0, S_{m}(0)>$ $0, I_{m}>0$ and $A_{m}(0)>0$ the solutions $\left(S_{f_{c}}(t), S_{f_{n}}(t), S_{f_{h}}(t), I_{f_{c}}(t), I_{f_{n}}(t), I_{f_{h}}(t), A_{f}(t), S_{m}(t), I_{m}(t)\right.$ and $\left.A_{m}(t)\right)$ of model system (5) are positively invariant for all $t>0$.

Proof. Let $t_{1}=\sup \left(t>0 \mid S_{f_{c}}>0, S_{f_{n}}>0, S_{f_{h}}>0, I_{f_{c}}>0, I_{f_{n}}>0, I_{f_{h}}>0, A_{f}>0, S_{m}>0, I_{m}>\right.$ $\left.0, A_{m}>0\right)$. From the first equation $S_{f_{c}}^{\prime}(t)=\delta_{1} \phi \Lambda-\left(\lambda_{f_{c}}(t)+\mu+\alpha\right) S_{f_{c}}(t)$ from which the integrating factor is given by $\exp \left\{\int_{0}^{t} \lambda_{f_{c}}(s) d s+(\alpha+\mu) t\right\}$. Multiplying the first equation by the integrating factor we obtain the following equation

$$
\frac{d}{d t}\left[S_{f_{c}}(t) \exp \left\{\int_{0}^{t} \lambda_{f_{c}}(s) d s+(\alpha+\mu) t\right\}\right]=\delta_{1} \phi \Lambda \exp \left\{\int_{0}^{t} \lambda_{f_{c}}(s) d s+(\alpha+\mu) t\right\} .
$$

Solving this equation, we obtain

$$
S_{f_{c}}(t) \exp \left\{\int_{0}^{t} \lambda_{f_{c}}(s) d s+(\alpha+\mu) t\right\}-S_{f_{c}}(0)=\int_{0}^{t} \delta_{1} \phi \Lambda \exp \left\{\int_{0}^{\tau} \lambda_{f_{c}}(p) d p+(\alpha+\mu) p\right\} d \tau
$$


from which we deduce that

$$
\begin{aligned}
S_{f_{c}}(t) & =S_{f_{c}}(0) \exp -\left\{\int_{0}^{t} \lambda_{f_{c}}(s) d s\right. \\
& +(\alpha+\mu) t\}+\exp -\left\{\int_{0}^{t} \lambda_{f_{c}}(s) d s+(\alpha+\mu) t\right\} \cdot \int_{0}^{t} \delta_{1} \phi \Lambda \exp \left\{\int_{0}^{\tau} \lambda_{f_{c}}(p) d p+(\alpha+\mu) p\right\} d \tau \geq 0
\end{aligned}
$$

Similarly, it can be shown that $\left(S_{f_{n}}(t)>0, S_{f_{h}}(t)>0, I_{f_{c}}(t)>0, I_{f_{n}}(t)>0, I_{f_{h}}(t)>0, A_{f}(t)>\right.$ $0, S_{m}(t)>0, I_{m}(t)>0, A_{m}(t)>0$.)

Theorem 2. The region $\mathcal{D}=\mathcal{W} \cup \mathcal{M} \subset \mathbb{R}_{+}^{7} \times \mathbb{R}_{+}^{3}$ is positively-invariant for model system (5) with non-negative initial conditions in $\mathbb{R}^{10}$.

Proof. We analyse model system (5) in a biologically-feasible region as follows. Consider the feasible region: $\mathcal{D}=\mathcal{W} \cup \mathcal{M} \subset \mathbb{R}_{+}^{7} \times \mathbb{R}_{+}^{3}$, where

$$
\mathcal{W}=\left\{S_{f_{c}}, S_{f_{n}}, S_{f_{h}}, I_{f_{c}}, I_{f_{n}}, I_{f_{h}}, A_{f} \in \mathbb{R}_{+}^{7}\right\}
$$

and

$$
\mathcal{M}=\left\{S_{m}, I_{m}, A_{m} \in \mathbb{R}_{+}^{3}\right\} .
$$

Summation of the first seven and the last three equations of model system (5) yields

$$
\begin{aligned}
& \phi \Lambda-\mu \mathcal{W}(t)-\alpha N_{f} t-\nu A_{f}(t), \\
& (1-\phi) \Lambda-\mu \mathcal{M}(t)-\alpha N_{m}(t)-\nu A_{m}(t) .
\end{aligned}
$$

Since

$\left(A_{f}(t) \leq N_{f}(t) \leq \mathcal{M}(t)\right)$, then from equation (9) it follows that

$$
\phi \Lambda-(\mu+\alpha+\nu) \mathcal{W}(t) \leq \frac{d \mathcal{W}(t)}{d t} \leq \phi \Lambda-\mu \mathcal{W}(t)
$$

Hence

$$
\frac{\phi \Lambda}{(\mu+\alpha+\nu)} \leq \liminf _{t \rightarrow \infty} \mathcal{W}(t) \leq \limsup _{t \rightarrow \infty} \mathcal{W}(t) \leq \frac{\phi \Lambda}{\mu}
$$

and therefore $\lim \sup _{t \rightarrow \infty} \mathcal{W}(t) \leq \frac{\phi \Lambda}{\mu}$. In a similar fashion, we can show that $\limsup _{t \rightarrow \infty} \mathcal{M}(t) \leq$ $\frac{(1-\phi) \Lambda}{\mu}$. Using a standard comparison theorem [42], it can be shown that

$\mathcal{W}(t) \leq \mathcal{W}(0) e^{-\mu t}+\frac{\phi \Lambda}{\mu}\left(1-e^{-\mu t}\right)$ and $\mathcal{M}(t) \leq \mathcal{M}(0) e^{-\mu t}+\frac{(1-\phi) \Lambda}{\mu}\left(1-e^{-\mu t}\right)$. Particularly $\mathcal{M}(t) \leq$ $\frac{\phi \Lambda}{\mu}$ if $\mathcal{W}(0) \leq \frac{\phi \Lambda}{\mu}$ and $\mathcal{M}(t) \leq \frac{(1-\phi) \Lambda}{\mu}$ if $\mathcal{M}(0) \leq \frac{(1-\phi) \Lambda}{\mu}$. The region $\mathcal{D}$ is thus positivelyinvariant and it is sufficient to consider the dynamics of the flow generated by model system $(5)$ in $\mathcal{D}$. In this region, the model can be considered as being epidemiologically and mathematically well-posed [29]. Thus, every solution to model system (5) with initial conditions in $\mathcal{D}$ remains in $\mathcal{D}$ for all $t>0$. 


\subsection{Disease-free equilibrium}

In order to analyse and understand the time evolution of an epidemic, typically, one studies the equilibria and their associated stability using an epidemic threshold value called the reproductive number. Setting the right hand side of model system (5) to zero, we obtain the disease-free equilibrium $\xi_{0}$ as

$$
\xi_{0}=\left(S_{f_{c}}^{0}, S_{f_{n}}^{0}, S_{f_{h}}^{0}, S_{m}^{0}, I_{f_{c}}^{0}, I_{f_{n}}^{0}, I_{f_{h}}^{0}, I_{m}^{0}, A_{f}^{0}, A_{m}^{0}\right)=\left(\frac{\delta_{1} \phi \Lambda}{(\alpha+\mu)}, \frac{\delta_{2} \phi \Lambda}{(\alpha+\mu)}, \frac{\delta_{3} \phi \Lambda}{(\alpha+\mu)}, \frac{(1-\phi) \Lambda}{(\alpha+\mu)}, 0,0,0,0,0,0\right) .
$$

We define the birth control induced reproductive number $\mathcal{R}_{H}$ as the expected number of secondary cases produced, in a completely susceptible population, by a typical infective individual when introduced into a population using condoms, traditional and HC methods. If $\mathcal{R}_{H}>1$ the disease will successfully invade a population and an epidemic occurs, while $\mathcal{R}_{H}<1$ precludes invasion. An associated threshold, $\mathcal{R}_{0}$ is defined as the basic reproductive number for the community in the absence of birth control. We use the approach in $[18,85]$ and the reproductive number $\mathcal{R}_{H}$ is defined as the spectral radius of the next generation matrix $F V^{-1}$ [85], from which we deduce the dominant eigen value $\mathcal{R}_{H}$,

$$
\mathcal{R}_{H}=\sqrt{\frac{c_{f} c_{m} \beta_{f} \beta_{m}\left[\rho_{m f_{c}}^{2}\left(1-p_{c}\right)^{2} \delta_{1}+\rho_{m f_{n}}^{2} \delta_{2}+\rho_{m f_{h}}^{2} \theta \chi \delta_{3}\right]}{(\alpha+\gamma+\mu)^{2}}}=\mathcal{R}_{0} \mathcal{H}
$$

The quantity $\mathcal{H}=\sqrt{\rho_{m f_{c}}^{2}\left(1-p_{c}\right)^{2} \delta_{1}+\rho_{m f_{n}}^{2} \delta_{2}+\rho_{m f_{h}}^{2} \theta \chi \delta_{3}}$ is the heterogeneity brought about by use of different birth control measures.

\subsection{Stability of disease-free equilibrium}

The following result follows from [85].

Lemma 1. The disease-free equilibrium of model system (5) is locally asymptotically stable if $\mathcal{R}_{H}<1$, and unstable if $\mathcal{R}_{H}>1$.

In Lemma 1 we stated that if $\mathcal{R}_{H}<1$, the fixed point $\xi_{0}$ is locally asymptotically stable. We now investigate the global asymptotically properties of the disease-free equilibrium.

Theorem 3. The disease-free equilibrium of model system (5) is globally asymptotically stable for $\mathcal{R}_{H}<1$ on $\mathcal{D}$.

Proof. Consider the Lyapunov function

$$
\mathcal{V}(t)=a_{1} I_{f_{c}}+b_{1} I_{f_{n}}+c_{1} I_{f_{h}}+d_{1} I_{m}
$$

where $a_{1}, b_{1}, c_{1}$ and $d_{1}$ are constants to be determined. The time derivative along the solution path of 
$\mathcal{V}(t)$ yields

$$
\frac{d}{d t} \mathcal{V}(t)=\left\{\begin{array}{l}
=\left[a_{1} \dot{I_{f_{c}}}+b_{1} \dot{I_{f_{n}}}+c_{1} \dot{I_{f_{h}}}+d_{1} \dot{I_{m}}\right] \\
=a_{1}\left[\rho_{m f_{c}}\left(1-p_{c}\right) c_{f} \beta_{m} \frac{I_{m}}{N_{m}} S_{f_{c}}-(\gamma+\mu+\alpha) I_{f_{c}}\right] \\
+b_{1}\left[\rho_{m f_{n}} c_{f} \beta_{m} \frac{I_{m}}{N_{m}} S_{f_{n}}-(\gamma+\mu+\alpha) I_{f_{n}}\right] \\
+c_{1}\left[\rho_{m f_{h}} \chi c_{f} \beta_{m} \frac{I_{m}}{N_{m}} S_{f_{h}}-(\gamma+\mu+\alpha) I_{f_{h}}\right] \\
+d_{1}\left[\rho_{m f_{c}}\left(1-p_{c}\right) c_{m} \beta_{f} \frac{I_{f_{c}}}{N_{f}} S_{m}+\rho_{m f_{n}} c_{m} \beta_{f} \frac{I_{f_{n}}}{N_{f}} S_{m}+\rho_{m f_{h}} \theta c_{m} \beta_{f} \frac{I_{f_{h}}}{N_{f}} S_{m}-(\gamma+\mu+\alpha) I_{m}\right] \\
=\left[a_{1} \rho_{m f_{c}}\left(1-p_{c}\right) c_{f} \beta_{m} \frac{S_{f_{c}}}{N_{m}}+b_{1} \rho_{m f_{n}} c_{f} \beta_{m} \frac{S_{f_{n}}}{N_{m}}+c_{1} \rho_{m f_{h}} \chi c_{f} \beta_{m} \frac{S_{f_{h}}}{N_{m}}-d_{1}(\gamma+\alpha+\mu)\right] I_{m} \\
+\left[d_{1} \rho_{m f_{c}}\left(1-p_{c}\right) c_{m} \beta_{f} \frac{S_{m}}{N_{f}}-a_{1}(\gamma+\alpha+\mu)\right] I_{f_{c}} \\
+\left[d_{1} \rho_{m f_{n}} c_{m} \beta_{f} \frac{S_{m}}{N_{f}}-b_{1}(\gamma+\alpha+\mu)\right] I_{f_{n}} \\
+\left[d_{1} \rho_{m f_{h}} \theta c_{m} \beta_{f} \frac{S_{m}}{N_{f}}-c_{1}(\gamma+\alpha+\mu)\right] I_{f_{h}}, \\
\leq\left[a_{1} \rho_{m f_{c}}\left(1-p_{c}\right) c_{f} \beta_{m} S_{f_{c}}+b_{1} \rho_{m f_{n}} c_{f} \beta_{m} S_{f_{n}}+c_{1} \rho_{m f_{h}} \chi c_{f} \beta_{m} S_{f_{h}}-d_{1}(\gamma+\alpha+\mu)\right] I_{m} \\
+\left[d_{1} \rho_{m f_{c}}\left(1-p_{c}\right) c_{m} \beta_{f} S_{m}-a_{1}(\gamma+\alpha+\mu)\right] I_{f_{c}} \\
+\left[d_{1} \rho_{m f_{n}} c_{m} \beta_{f} S_{m}-b_{1}(\gamma+\alpha+\mu)\right] I_{f_{n}} \\
+\left[d_{1} \rho_{m f_{h}} \theta c_{m} \beta_{f} S_{m}-c_{1}(\gamma+\alpha+\mu)\right] I_{f_{h}} .
\end{array}\right.
$$

Setting the coefficients of $I_{f_{c}}, I_{f_{n}}$ and $I_{f_{h}}$ to zero and solving for $b_{1}, c_{1}$ and $d_{1}$ we obtain

$$
\left.\begin{array}{rl}
b_{1} & =\frac{a_{1} \rho_{m f_{n}}}{\left(1-p_{c}\right) \rho_{m f_{c}}} \\
c_{1} & =\frac{\theta a_{1} \rho_{m f_{h}}}{\left(1-p_{c}\right) \rho_{m f_{c}}} \\
d_{1} & =\frac{(\alpha+\gamma+\mu) a_{1}}{c_{m}\left(1-p_{c}\right) S_{m} \beta_{f} \rho_{m f_{c}}}
\end{array}\right\} .
$$

Substituting for the constants and equilibrium relations of $S_{f_{c}}, S_{f_{n}}, S_{f_{h}}$ and $S_{m}$ into equation (15), we obtain

$$
\begin{aligned}
\frac{d}{d t} \mathcal{V}(t) & \leq \frac{a_{1}}{(1-p) \rho_{m f_{c}}}\left[-\frac{(\alpha+\mu)(\alpha+\gamma+\mu)^{2}}{\Lambda(1-\phi) c_{m} \beta_{f}}+\frac{\Lambda \phi c_{f} \beta_{m}\left((1-p)^{2} \delta_{1} \rho_{m f_{c}}^{2}+\theta \chi \delta_{3} \rho_{m f_{h}}^{2}+\delta_{2} \rho_{m f_{n}}^{2}\right)}{(\alpha+\mu)}\right] I_{m} \\
& \leq \frac{a_{1}}{(1-p) \rho_{m f_{c}}} \frac{(\alpha+\gamma+\mu)}{c_{m} \beta_{f}}\left[\mathcal{R}_{H}^{2}-1\right] I_{m} \\
& \leq 0, i f \mathcal{R}_{H}<1,
\end{aligned}
$$

for the relation $\frac{N_{m}}{N_{f}}=\frac{(1-\phi)}{\phi}$ which is valid at the disease free equilibrium. Using the Lyapunov stability theorem $\frac{d}{d t} \mathcal{V}(t)$ is negative definite. The omega limit set of each solution is the largest invariant set for which $S_{f_{c}}=S_{f_{c}}^{*}, S_{f_{n}}=S_{f_{n}}^{*}, S_{f_{h}}=S_{f_{h}}^{*}, S_{m}=S_{m}^{*}, I_{f_{c}}=0, I_{f_{n}}=0, I_{f_{h}}=0, I_{m}=0$ for which $\mathcal{E}_{0}$ is a singleton. By LaSalle's invariance principle [44], the disease-free equilibrium $\mathcal{E}_{0}$ is globally asymptotically stable in $\mathcal{D}$. This achieves the proof. 
By Theorem 3 if on average every individual infects less than one other individual then they fail to replenish themselves and disease prevalence will be a decreasing function of $I_{i}(\mathrm{t})$ and the disease dies out regardless of the initial levels of the epidemic. Global stability of the disease-free equilibrium implies that if $\mathcal{R}_{H}<1$, HIV will be eliminated from the community.

\subsection{Endemic equilibrium}

Model system (5) has an endemic equilibrium point derived in terms of the force of infection given by,

$$
\xi^{*}=\left\{\begin{array}{l}
S_{f_{c}}^{*}=\frac{\Lambda \phi \delta_{1}}{\left(\mathcal{K}_{2}+\lambda_{f_{c}}^{*}\right)}, S_{f_{h}}^{*}=\frac{\Lambda \phi \delta_{2}}{\left(\mathcal{K}_{2}+\lambda_{f_{n}}^{*}\right)}, S_{f_{n}}^{*}=\frac{\Lambda \phi \delta_{3}}{\left(\mathcal{K}_{2}+\lambda_{f_{h}}^{*}\right)}, S_{m}^{*}=\frac{\Lambda(1-\phi)}{\left(\mathcal{K}_{2}+\lambda_{m}^{*}\right)} \\
I_{f_{c}}^{*}=\frac{\Lambda \phi \delta_{1} \lambda_{f_{c}}^{*}}{\mathcal{K}_{1}\left(\mathcal{K}_{2}+\lambda_{f_{c}}^{*}\right)}, I_{f_{n}}^{*}=\frac{\Lambda \phi \delta_{2} \lambda_{f_{n}}^{*}}{\mathcal{K}_{1}\left(\mathcal{K}_{2}+\lambda_{f_{n}}^{*}\right)}, \\
I_{f_{h}}^{*}=\frac{\Lambda \phi \delta_{3} \lambda_{f_{h}}^{*}}{\mathcal{K}_{1}\left(\mathcal{K}_{2}+\lambda_{f_{h}}^{*}\right)}, I_{m}^{*}=\frac{\Lambda(1-\phi) \lambda_{m}^{*}}{\mathcal{K}_{1}\left(\mathcal{K}_{2}+\lambda_{m}^{*}\right)}
\end{array}\right.
$$

where $\mathcal{K}_{1}=(\alpha+\gamma+\mu)$ and $\mathcal{K}_{2}=(\alpha+\mu)$ with

$$
\begin{aligned}
& \lambda_{m}^{*}=\beta_{f} c_{m} \frac{\rho_{m f_{c}}\left(1-p_{c}\right) I_{f_{c}}^{*}+\rho_{m f_{n}} I_{f_{n}}^{*}+\rho_{m f_{h}} \theta I_{f_{h}}^{*}}{N_{f}^{*}}, \lambda_{f_{c}}^{*}=\rho_{f_{c} m}\left(1-p_{c}\right) c_{f} \beta_{m} \frac{I_{m}^{*}}{N_{m}^{*}}, \\
& \lambda_{f_{n}}^{*}=\rho_{f_{n} m} c_{f} \beta_{m} \frac{I_{m}^{*}}{N_{m}^{*}} \text { and } \lambda_{f_{h}}^{*}=\rho_{f_{h} m} \chi c_{f} \beta_{m} \frac{I_{m}^{*}}{N_{m}^{*}} .
\end{aligned}
$$

After several algebraic manipulations shown in Appendix A, we obtain, a quatic polynomial $f\left(\lambda_{m}^{*}\right)$ expressed in terms of $\lambda_{m}^{*}$ as follows

$$
f\left(\lambda_{m}^{*}\right)=\mathcal{A}_{1} \lambda_{m}^{* 4}+\mathcal{A}_{2} \lambda_{m}^{* 3}+\mathcal{A}_{3} \lambda_{m}^{* 2}+\mathcal{A}_{4} \lambda_{m}^{*}
$$

from which the following solutions are obtained,

$$
\begin{aligned}
& \lambda_{m}^{*}=0, \\
& \lambda_{m}^{*}=\frac{\frac{2^{4 / 3} \mathcal{A}_{5}}{\left(\sqrt{\mathcal{A}_{6}^{2}-4 \mathcal{A}_{5}^{3}}-\mathcal{A}_{6}\right)^{1 / 3}}+2^{2 / 3}\left(\sqrt{\left(\mathcal{A}_{6}^{2}-4 \mathcal{A}_{5}^{3}\right)^{1 / 3}}-\mathcal{A}_{6}\right)-2 \mathcal{A}_{2}}{6 \mathcal{A}_{1}}, \\
& \lambda_{m}^{*}=\frac{\frac{2^{7 / 3} \mathcal{A}_{5}}{\left(\sqrt{\mathcal{A}_{6}^{2}-4 \mathcal{A}_{5}^{3}}-\mathcal{A}_{6}\right)^{1 / 3}}+{\sqrt{\mathcal{A}_{6}^{2}-4 \mathcal{A}_{5}^{3}}}^{1 / 3}-4 \mathcal{A}_{2}}{12 \mathcal{A}_{1}}, \\
& \lambda_{m}^{*}=\frac{\frac{2^{7 / 3} \mathcal{A}_{5}}{\left(\sqrt{\mathcal{A}_{6}^{2}-4 \mathcal{A}_{5}^{3}}-\mathcal{A}_{6}\right)^{1 / 3}}-\sqrt{\mathcal{A}_{6}^{2}-4 \mathcal{A}_{5}^{3}}{ }^{1 / 3}-4 \mathcal{A}_{2}}{12 \mathcal{A}_{1}},
\end{aligned}
$$

with

$$
\left\{\begin{array}{l}
\mathcal{A}_{5}=\mathcal{A}_{2}^{2}-3 \mathcal{A}_{1} \mathcal{A}_{3} \\
\mathcal{A}_{6}=2 \mathcal{A}_{2}^{3}-9 \mathcal{A}_{1} \mathcal{A}_{2} \mathcal{A}_{3}+27 \mathcal{A}_{1}^{2} \mathcal{A}_{4}
\end{array}\right.
$$


Equation (18) represents model system (5) at the disease-free equilibrium whose qualitative properties have already been resolved in Sections 3.1. In order to show existence of endemic equilibria for model system (5) it is necessary that we show that one of the solutions to $\lambda_{m}^{*}$ in equations $(19,20,21)$ is positive for the condition $\mathcal{R}_{H}>1$ but that exercise is a laborious task. Instead using parameter values in Table 2 we plot the graphs of $\lambda_{m}^{*}$ in equations $(19,20,21)$ to show existence of endemic equilibria. The plot of $\lambda_{m}^{*}$ in equation (19) is shown in Figure 2 and depicts an epidemic or forward bifurcation curve [19]. The graph of $\lambda_{m}^{*}$ in equations $(20,21)$ exists in the negative octant of $\lambda_{m}^{*}$ and denotes imaginary populations that are not biologically feasible and therefore of no interest to us. From Figure 2 we deduce the following lemma:

Lemma 2. Model system (5) has a unique endemic equilibrium which exists for $\mathcal{R}_{H}>1$ and does not exist otherwise.

Consistent with standard results in HIV models, the forward bifurcation curve has, no positive endemic equilibria for $\mathcal{R}_{H}<1$ and a low level of endemicity when $\mathcal{R}_{H}$ is slightly above 1 [19]. In our previous work, [47] we showed that $\mathcal{R}_{H}>\sqrt{2}$ was sufficient condition (which is more burdensome than $\mathcal{R}_{H}>1$ ) for HIV endemicity. The implication from that result and result from Figure 2 is that at $\mathcal{R}_{H}=1$ disease control is feasible and this is important result for resource constrained communities such as exist in subSaharan Africa. In Figure 2, the black bold line represents a stable disease-free equilibrium for $\mathcal{R}_{H}<1$, and the blue line represents a stable endemic equilibrium point when $\mathcal{R}_{H}>1$, with low endemicity noted for $\mathcal{R}_{H}$ close to 1 . In the event that the disease-free equilibrium exists for $\mathcal{R}_{H}>1$, then it is a saddle and is depicted by the red broken line. Away from the vicinity of $\mathcal{R}_{H}=1$, the epidemic size is an increasing function of the birth control induced reproductive number hence a forward bifurcation manifests. The same process to show the existence of the endemic equilibrium point can be repeated by analysing the

conditions of existence for $\lambda_{f_{c}}^{*}, \lambda_{f_{n}}^{*}$ and $\lambda_{f_{h}}^{*}$. It is straight forward to show the local stability of the endemic equilibrium using the center manifold theorem [46,47].

\subsection{Global stability of the endemic equilibrium}

Results in Lemma 2 suggest the existence of an endemic equilibrium point $\xi^{*}$ which destabilises the disease-free equilibrium $\xi_{0}$ and is locally stable whenever $\mathcal{R}_{H}>1$. In Theorem 4 we state conditions for its global stability and the proof is given in Appendix B.

Theorem 4. If $\mathcal{R}_{H}>1$, the endemic equilibrium state $\xi^{*} \in \mathcal{D}$ exists and is globally asymptotically stable in $\mathcal{D}$ if

$$
S_{f_{c}}^{*} I_{f_{c}}=S_{f_{c}} I_{f_{c}}^{*}, S_{f_{n}}^{*} I_{f_{n}}=S_{f_{n}} I_{f_{n}}^{*}, S_{f_{h}}^{*} I_{f_{h}}=S_{f_{h}} I_{f_{h}}^{*}, S_{m}^{*} I_{m}=S_{m} I_{m}^{*}
$$

Serious implications regarding HIV/AIDS dynamics emanate from the global stability of the endemic equilibrium. For $\mathcal{R}_{H}>1$, the implication is that the disease persists irrespective of the initial conditions. The onerous task for policy makers therefore is the reduction of the reproductive number to below unit in order to destabilise the endemic equilibrium and control the epidemic.

\section{Numerical analysis and results}

In order to explore qualitative features of our model through numerical simulations, we used data from sub-Saharan Africa. Transmission parameters are key components in any HIV modeling study. Heterosexual HIV transmission is estimated to have a very low probability [22], but due to confounding effects of cofactors that differ, it varies within regions. Cuadros et al [15] showed that a single value for HIV infectivity is inappropriate and fails to describe the dynamics of the epidemic in sub-Saharan Africa. 
They concluded that the inclusion of individual variation generated by transient yet repeated increases in HIV viral load associated with co-infections may provide a biological basis for the widespread and accelerated spread of HIV in sub-Saharan Africa. Further, results in [5] showed that the proportion of men reporting that they have had 2 or more sex partners in a year ranged from $1 \%$ to $28 \%$ in sub-Saharan Africa. In other studies, [74] it was shown that the mean number of lifetime sexual partners ranged from 3.4 to 12.9 with a mean of 6.3 in sexually active men in the region. Mean lifetime number of partners of men in the immediate local community was predictive of hazard of HIV acquisition in women. Recent evidence suggests that the adult HIV-1 latent period may be shorter in some sub-Saharan African populations than in Western populations [22] and we assumed an average incubation period of between 4 to 10 years. A Runge-Kutta algorithm of order 4 coded in Matlab is used to carry out numerical simulations to illustrate some of the analytical results given in this paper, to investigate some qualitative features of our HIV/AIDS model, determine the effect of different parameters on the model and to fit our model to HIV prevalence data for Zimbabwe. We carried out sensitivity analysis on model system (5) to determine important model parameters using baseline values picked from HIV literature given in Table 2.

\subsection{Sensitivity analysis}

Parameter values used for model system (5) are estimates from different studies and therefore are unreliable since their actual values are not known. Sensitivity analysis is necessary because of the structural complexity of the model which is coupled with lack of certainty when estimating input parameters [6]. To account for this uncertainty in parameter input values, we use a statistical analysis technique (Latina Hypercube Sampling (LHS)) that allows simultaneous random variation of all the input parameters [48]. The range is divided into $n$ intervals each with equal chance. Each random variable is then assigned a probability density function. Thus there are $n$ observations for each of the $k$ parameters [53]. For one sample point $x_{i}$, a random selection is made on $x_{1}$ and paired with a random selection on $x_{2}, x_{3} \ldots x_{k}$ and this constitutes the first sample point. The process is repeated $n$ times to give the first sample. A sensitivity analysis may then be performed by calculating partial rank correlation coefficients (PRCC) for each input parameter (sampled by the LHS scheme) and each outcome variable [6]. A PRCC quantifies the degree of influence of each parameter on each response while holding the influence of other parameters constant. A monotonic relationship between the response variables and the model parameters is assumed [32] in PRCC analysis. A PRCC is bounded between -1 and 1. Values near the magnitude 1 indicate a dominant parameter in influencing the response while the least important rank parameters are close to zero.

The PRCC analysis requires a monotonically increasing function [32], hence our use of the combined AIDS cases $A,\left(A=A_{f}+A_{m}\right)$ to find the dominant input parameters. It is possible though that certain parameters might exhibit non-monotonic relationships with the output variable and consequently have a low PRCC but may produce huge changes in the outcome variable [6] and different model structures will give different sensitivity analysis results and similar PRCC results may not be obtained from other model structures. Parameters unrelated to the disease were excluded from the analysis that is the natural birth and death rates and the emigration rate. The baseline values associated with the response variable $A$ are tabulated in Table 2 and these parameters are assumed to be independent and come from a uniform distribution. The uniform distribution is useful when only the minimum and maximum range of a parameter is estimable [8] or when there is no evidence that the end of the ranges are less probable than the middle values [53]. If evidence points to the later, then a triangular distribution is more appropriate [8]. We used the observations from the LHS to calculate PRCCs between the response variable $A$ and fourteen constituent parameters of the birth control induced reproductive number. Scatter plots were generated and studied to check that the assumption of monotonicity was satisfied and some of these are shown in Figure 3. The PRCCs are shown in Table 1. 
In Table 1 the most influential parameters on the number of AIDS cases are those with $p<0.001$. These are $(i)$ the progression rate to AIDS $\gamma,(i i)$ the increased infectivity of females who use HCs $\theta$, (iii) the male partnership acquisition parameter $c_{m},(i v)$ increased susceptibility of females due to contraceptive use $\chi$, and $(v)$ the mutually accepted rate of sex between males and females who use HCs $\rho_{m_{f_{h}}}$. Three of these parameters are related to $\mathrm{HC}$ use. The increased infectivity of females who use contraceptives $(\theta)$ as birth control mechanism, has the greatest influence on the number of AIDS cases followed by the number of male partnerships. The parameter that represents increased susceptibility due to contraceptive use is next in importance followed by the mutual rate of sex between males and females that use HCs. The magnitude of the PRCC for male and female infectivity relative to the PRCC values of the other parameters related to HCs suggests that biological infectivity $\left(\beta_{f}, \beta_{m}\right)$ is of lesser influence in determining the number of AIDS cases a result which does not emphasise standard results and results in $[7,47,53]$ but emphasises the importance of parameters related to birth control. The mutual rate of sex between men and contraceptive users plays a very significant role (as does $\beta_{f}$ and $c_{f}$ ) as shown by the size of the PRCC. If contraceptive users become more preferable to men than condom users (may be through sensation seeking), more AIDS cases will manifest. The uncertainties in estimating the values of the parameters described above $\left(\theta, \chi, c_{m}, \rho_{f_{h} m}, \beta_{f}, \beta_{m}, c_{f}\right)$, are the most critical in contributing to the prediction precision (imprecision) of the number of HIV cases.

Sensitivity analysis is important in that it focuses data collection efforts [6] through identification of the critical parameters (due to their estimation uncertainty) in the prediction precision (imprecision) of future AIDS cases. The qualitative relationship between the input parameters and output variables is shown by the signs of the PRCCs. The negative signs indicate that an increase in that input parameter would result in the reduction of future AIDS cases and for an increase in the input parameters whose PRCCs are positive, the reverse is analogous. The partial rank correlation coefficients that are shown in Table 1 are plotted in Figure 4 and show qualitatively, the magnitude of the influence of the various parameters in $\mathcal{R}_{H}$ on the combined number of AIDS cases. We use a cut-off of $p<0.05$ for statistical significance of parameters on the model output. In Table 4 the PRCC for the parameter $p_{c}$ representing condom use and parameters representing population proportions using particular birth control methods $\left(\delta_{1}, \delta_{2}\right.$, and $\left.\delta_{3}\right)$ and the mutual rate of sex between men and women who use condoms and natural methods, are very low showing that these parameters are not significant in the outcome. However these PRCCs have $p>0.05$, showing that it cannot be ruled out that chance factors are determining the relationship.

A description of parameters, their baseline values and fitted values of parameters with significant PRCCs from Table 1 assuming no increased susceptibility of infectivity due HCs is given in Table 2. Using values in Table 2, we showed the qualitative relationship between the birth control induced reproductive number to some of its constituent components.

Figures $5(a)$ and $5(b)$ show that use of condoms and efficacy will reduce $\mathcal{R}_{H}$ and also HIV prevalence amongst users of barrier based methods of contraception. Condom efficacy has already been proven to be very high $(\approx 85 \%)$, and thus the only effort will be on scaling up its use. An increase in disease susceptibility and infectivity through use of HCs will result in higher values of $\mathcal{R}_{H}$ and hence disease prevalence as shown by Figures $5(c)$ and $5(d)$.

\subsection{Model fitting and predictions}

In order to assess the potential impact of using HCs on HIV dynamics, we fit our model to Zimbabwe HIV prevalence data. The first reported case of AIDS in Zimbabwe occurred in 1985. By the end of the decade, although data was sparse, an estimated $10 \%$ of the adult population were thought to be 
infected with HIV [80]. In the first half of the 1990s, prevalence rose drastically, peaking at $28.5 \%$ in 1997 [83]. Therein, the HIV prevalence began to decline and the adult prevalence rate stood at $23.7 \%$ in 2001 subsequently falling to $14.3 \%$ in 2010 [83]. A number of reasons have been proposed for this decline including economic meltdown which resulted in the outward migration of infected persons to seek medication outside the country. Using the current UNAIDS definition of prevalence $\frac{I_{i}+A_{i}}{S_{i}+I_{i}+A_{i}}$, the effect of AIDS deaths on prevalence is twofold. High AIDS deaths will either reduce prevalence or balance off new birth rates thereby maintaining high previous prevalence rates. We address this problem by removing AIDS cases from the numerator and denominator in our definition of prevalence and this is consistent with our previous assumption that individuals in the AIDS category are non interactive and therefore are unlikely to be observed using the current method which employees ante-natal clinics (although UNAIDS data is readjusted to include AIDS cases). Secondly, high AIDS related deaths are also thought to have resulted in reduced infectivity as people witnessed firsthand, the death of close relatives and friends and this resulted in behaviour changes [23] leading to the reduction of incidence rates which in turn would lower prevalence levels. Condom use has increased, and greater numbers are now delaying sexual debutancy and reduction of partnership has been observed since 2005 [80]. At the start of the epidemic, people that have lots of sex, are more likely to be infected quickly, while those that have infrequent sex, might never be infected. For this reason, in our data fit we include behaviour change and allow for a declining force of infection as the prevalence increases. As more people become symptomatic and visible to society, at risk individuals tend to acquire greater HIV awareness, and we thus make the force of infection responsive to this reduced susceptibility and make it a function of the symptomatic rate $(\gamma)$ so that the contact rate function is modelled by

$$
c_{i} \beta_{j} e^{-\varpi \gamma P}
$$

where the parameter $\varpi$ is the response due to reducing susceptibility and due to experiential learning resulting from people seeing individuals develop AIDS symptoms and $P$ is the prevalence function. Such modeling technique is used for data fitting in [31] but the authors use the number of AIDS deaths where we have used prevalence.

UNAIDS reported HIV prevalence data from the year 1990 onwards and this marks our initial modeling year. For the years 2008 and 2009, we used data estimates from MoHCW [91] with the rest coming from UNAIDS [76]. At the start of the epidemic in 1990 we set our initial sub-population levels to depict a prevalence level of $14 \%$ reported by UNAIDS [80] as at 1990. We employed least squares curve fitting function in Matlab to fit our model to HIV prevalence data for Zimbabwe. In the fitting we estimated, probabilities of HIV transmission $\left(\beta_{f}, \beta_{m}\right)$, response for behaviour change $(\varpi)$, incubation period $\gamma$ and emigration rate $(\alpha)$ and the estimated values are given in Table 2. Prevalence gives us the proportion of infected persons in the population and together with the index of people living with HIV and AIDS allows for forward planning. It however falls short in that it is affected by multiple factors such as progression to AIDS and outward migration or high AIDS death rates balancing off new birth rates and therefore maintaining high prevalence rates. Nowadays treatment may result in longevity of life while infected and therefore lead to higher prevalence rates. More often for a better understanding of the epidemic path, it is more insightful to obtain the actual number of new infections per unit time.

Plots of the prevalence and incidence functions are given in Figures $6(a)$ and $6(b)$. Assuming the same conditions persist, we make projections for prevalence and incidence up to the year 2020 and these are shown in Figures $6(c)$ and $6(d)$. The projections suggest that incidence curve will plateau below 0.01 from the year 2007. To estimate the potential impact of using HCs on HIV prevalence, we considered a scenario without $\mathrm{HC}$ use (i.e. $\theta=\chi=1$ ) and introduced their use in the year 2010. Using parameters estimated from the best fit in Figure $6(a)$ shown in Table 2, we projected HIV prevalence for different susceptibility and infectivity levels due to $\mathrm{HC}$ use and also explored the impact of using condoms for 
optimistic scenarios (in Figure 7).

Results in Figure $7(a)$ show that projected HIV prevalence will increase by $25 \%, 50 \%$ and $100 \%$ from the baseline epidemic levels when baseline values for susceptibility and infectivity $(\chi, \theta)$ are simultaneously increased by 2,3 , and 4 fold respectively. Thus a potential increase in susceptibility and infectivity due to HCs use has the capacity to increase HIV prevalence even in countries reporting plausible declines in HIV prevalence such as Zimbabwe. Model projections in Figures $7(b)$ and $(c)$ show the effects of increasing female infectivity and susceptibility on HIV prevalence respectively due to HC. However if $\mathrm{HC}$ use result in increased susceptibility only without commensurate increases in risk of infectivity to men, disease prevalence would be high in the cohort of $\mathrm{HC}$ users but will remain unchanged in the general population since contraceptive use is still limited to a small group of women in sub-Saharan Africa. This scenario removes the bridging effect of men between the high-risk core group and low-risk general population. Figure $7(d)$ shows that HIV prevalence would decrease by approximately $600 \%$ at a very optimistic consistency of condoms use of $100 \%$ for birth control. Thus an opportunity exists for HIV control through the use of condoms which save the dual purpose of birth control and HIV transmission.

\section{Discussion and conclusion}

While a straightforward causal link between HC use and heightened HIV transmission remains to be demonstrated, preliminary evidence suggesting potential increased risk to HIV susceptibility and infectivity due to $\mathrm{HC}$ use, $[3,17,28,40,51,55,70]$, compels for the modelling of theoretical aspects and the what if scenarios regarding the dynamic influence of $\mathrm{HC}$ use on HIV transmission. We designed a deterministic compartmental model to provide perspectives on the dynamical interaction of HIV and the social and biological aspects of reproductive health practiced by communities in sub-Saharan Africa. In order to ensure that our model is at the confluence of theory and the real world, and provide both abstractive and biological meaning, we fitted our model to HIV data for Zimbabwe and used the parameters that gave the best fit to predict the implications of HC use on HIV prevalence.

Our results demonstrated the potential of HC use to compound the HIV epidemic. The model was designed to include possible increases in susceptibility and infectivity due to HC use. Numerical results showed that infectivity of women on HC may play a disproportionately prominent role in driving HIV dynamics (Figure $7(b)$ ). Depo-Provera users may cause heightened transmission to their male partners who in turn act as a bridge and pass the virus on to the lower risk group of women. The more partners males have, the more influential they become in driving the epidemic and the more $\mathrm{HC}$ use becomes important in shaping HIV transmission dynamics. This result on male partner acquisition rate is standard [47], but the result on infectivity suggesting heterosexual male infectivity to be less influential than female infectivity in a model that incorporates birth control is novel. In other words $\mathrm{HC}$ use causes female infectivity to become more significant in determining disease dynamics over male infectivity. Several studies have shown male-female infectivity exceed female-male infectivity per each coital act [49,64], and these results have been used to explain contrasting gender differentials in HIV prevalence. Our results show that when HCs are used, female to male infectivity of HC users taken together with male partnership formation rates, would become the key driver of the epidemic. Low HIV endemicity is noted for the cohort that uses condoms for birth control, but overall condom use is shown to be insufficient in controlling the epidemic if other birth control methods are employed by women. However if condoms are universally used as a birth control tool, HIV control is feasible. This result is consistent with results in previous studies $[1,53,68]$.

We fitted our model to prevalence data from Zimbabwe extracted from UNAIDS [76] and MoHCW [91] from the years 1990 to 2010 (Figure 6 (a)). To estimate the potential impact of using HC on HIV prevalence, we considered a scenario without HC use and introduced their use in the year 2010. When 
susceptibility and infectivity were increased simultaneously from the baseline case, the results suggest that HIV prevalence in the year 2020 would increase by $25 \%, 50 \%$ and $100 \%$ respectively showing that the potential increase of the use of DMPA would enlarge the epidemic and derail disease control efforts (Figure $7(a)$ ). It is important to note that data used in the data fit spans 20 years. Substantial epidemiological evolution would have taken place during that long time span including changes in susceptibility and parameter values. We replaced the constant transmission coefficient with an exponentially decaying function in order to reflect this reduction in susceptibility. However other aspects of the epidemiology of HIV/AIDS such as treatment which affects the longevity of the infectivity period are salient features of the fitted data but are not captured in the model. Certainly the HIV adjusted expected lifetime $\gamma$ at the start of the epidemic cannot be expected to be the same after the point of introduction of HIV treatment and neither can be, the infectivity of individuals reported to be infected. The peak of the HIV epidemic coincided with a severe economic recession with estimated range of more than 3 million people to a few hundred thousand [21] having fled the economic meltdown that caused severe food shortages and a collapse of the health system. In recent times, the economy has stabilised and free treatment is provided locally by government agencies and migration for purposes of treatment is no longer a necessity in Zimbabwe. However our model does have a parameter to capture outward migration of infectives. Consequently, the parameters that give the best model fit are, infact, a smoothed average of different facets characterising the epidemic through the period of data collection.

Our results suggest that while biological aspects about the disease itself, that is transmission related parameters, play a significant role in influencing disease dynamics, they play a relegated role to parameters related to HC. Results from this study, although based on inconclusive evidence of the scientific role of DMPA as a cause for accelerated HIV transmissions, reinforce the need for more effort and resources to be put into further research to assess the potential impact of HC methods of birth control on HIV transmission and the need for guidelines for women using family planning services in HIV endemic settings. It is shown in this study that use of condoms for birth control, and protection against HIV in general [53,60], will result in lesser efforts being needed to fight the HIV epidemic (Figure $7(d)$ ). Condemnation and limited use of $\mathrm{HC}$ which are cheap and easy to self administer would probably cause increased maternal mortality and morbidity [28] and thus public health policy makers are already advocating the reorientation of family planning programmes [35] with a view of promoting condoms whose independent use has been shown to be effective at fighting HIV spread.

Our work is based on conflicting scientific evidence and must be upheld in a theoretical contextual framework. Until a definite causal relationship between HC use and heightened HIV transmission is established, recommendations by WHO advocating for individuals most at risk of HIV infection, to use dual protection with condoms, should be taken seriously. Only then, in light of our modelling results, can the specific transmission risk to men be highlighted together with their consequential potential to spread the disease to the rest of the low risk general population.

\section{Acknowledgments}

NJM and SDH-M acknowledges, with thanks, financial support from the National University of Science and Technology. The authors acknowledge Dr Christinah Chiyaka for useful comments and discussions on the manuscript.

\section{References}

[1] Ahmed S. et al, HIV incidence and sexually transmitted disease prevalence associated with condom use: a population study in Rakai, Uganda, AIDS, (15), 2171-2179, (2001). 
[2] AFP, May 12 2011, New birth control method urged for developing world women, http://www. google.com-hostednews-afp-article, (accessed 21-05-2012).

[3] Baeten J. M., Benki S., Chohan V. et al., Hormonal contraceptive use, herpes simplex virus infection, and risk of HIV-1 acquisition among Kenyan women, AIDS, (21), 1771-1777, (2007).

[4] Bahamondes L., Trevisan M., Andrade L. et al., The effect upon the human vaginal histology of the long-term use of the injectable contraceptive Depo-Provera, Contraception, (62), 23-27, (2000).

[5] Bingenheimer J. B., Men's multiple sexual partnerships in 15 Sub-Saharan African countries: Sociode-mographic patterns and implications, Studies in Family Planning, (41), 1-17, (2010).

[6] Blower S. M. and Dowlatabadi H., Sensitivity and uncertainty analysis of complex models of disease transmission: an HIV model as an example, International Statistical Review, (62), 229-243, (1994).

[7] Blower S. M., Hartel D., Dowlatabadi H., Anderson R. M. and May R. M., Drugs, Sex and HIV: A Mathematical Model for New York City Philosophical Transactions of the Royal Society of London, (331), 171-187, (1991).

[8] Blower S. M. and Sanchez M. A., Uncertainty and sensitivity analysis of the basic reproductive rate: Tubercolosis as an example, American Journal of Epidemiology, (145), 1127-37, (1997).

[9] Blythe P.S. and Castillo-Chavez C., Like with Like preference and sexual mixing models, Mathematical Biosciences, 96, 221-238 (1989).

[10] Brauer F., Epidemic models with heterogeneous mixing and treatment, Bulletin of Mathematical Biology, (70), 1869-1885,(2008).

[11] Busenserg S. and Chavez C.C., A general solution of the problem of mixing of subpopulations and its application to risk, and age structured epidemic models of the spread of AIDS, IMA Journal of Mathematics Applied in Medicine and Biology, 8, 1-29, (1991).

[12] Catholic Church contraception policy, http://dbp.idebate.org-en-index.php-Debate:-Catholic-Church-contraception-policy, (accessed 15-12-2012).

[13] Contraception-Ancient methods in use today, Evolution of the condom.

www.scienceclarified.com/Ci-Co/Contraception.html, (accessed 21-06-2012).

[14] Creanga A. A., Gillespie D., Karklins S. and Tsui A. O., Low contraceptive use among the poor in Africa: An equity issue, urlhttp://www.who.int/bulletin/volumes/89/4/10-083329/en/index.html, (accessed 11-01-2013).

[15] Cuadros D. F., Crowley P. H., Augustine B., Stewart S. L. and Garcáa-Ramos G., Effect of variable transmission rate on the dynamics of HIV in sub-Saharan Africa, BMC Infectious Diseases, (11), 216, (2011).

[16] Del Valle S. Y., Hyman J. M., Hethcote C. and Eubank S. G., Mixing patterns between age groups in social networks, Social Networks, 29, 539-554, (2007).

[17] Delvaux T. and Buv A., Hormonal contraception and HIV acquisition - what is the evidence? What are the policy and operational implications?, European Journal of Contraception and Reproductive, 18(1), 15-26, (2013). 
[18] Diekmann O., Heesterbeek J.A.P. and Metz J.A.P., On the definition and computation of the basic reproduction ratio $R_{0}$ in models for infectious diseases in heterogeneous populations, Journal of Mathematical Biology, (28) 365-382, (1990).

[19] Dushoff J., Wenzhang H. W. and Castillo-Chavez C., Backwards bifurcations and catastrophe in simple models of fatal diseases, Journal of Mathematical Biology, (36), 227-248, (1998).

[20] Fan M., Chowa L. and Feng Z., Dynamics of a multi-group epidemiological model with group-targeted vaccination strategies, Journal of Theoretical Biology, 291, 56-64, (2011).

[21] Global envision, The Effect of Migration on HIV Rates (2007), http://www.globalenvision.org/library/9/1758, (accessed 11-01-2013).

[22] Gregson S., Garnett G. P., Shakespeare R., Foster G. and Anderson R. M., Determinants of the demographic impact of HIV-1 in sub-Saharan Africa: the effect of a shorter mean adult incubation period on trends in orphanhood, Health Transition Review, AIDS Impact and Prevention in the Developing World: Demographic and Social Science Perspectives, (4), 65-92, (1994).

[23] Gregson S., et al. HIV Decline associated with behaviour change in eastern Zimbabwe, Science, 311 , 664-666, (2006).

[24] Gribble J. and Haffey J., Reproductive health in Sub-Saharan Africa, Population Reference Bureau, http://www.prb.org/pdf08/reproductivehealth-subsaharanafrica.pdf, (accessed 15/12/2012).

[25] Guardian, HIV could spread if birth control injections increase warn scientists: Researchers call for new guidelines for women using family planning in AIDS-hit areas,

(http://www .guardian.co.uk/world/2011/oct/04/hiv-spread-birth-control-warn-scientists, (accessed 21-05-2012).

[26] Haddad L. B., Contraceptive Methods and Risk of HIV Acquisition or Female to male Transmission, Current HIV/AIDS Reports, 11(4), 447-458, (2014).

[27] Hansasuta P. and Rowland-Jones S. L., The changing face of HIV and AIDS HIV-1 transmission and acute HIV-1 infection, British Medical Bulletin, (58), 109-127, (2001).

[28] Heffron R., Donnell D., Rees H., et al. Use of hormonal contraceptives and risk of HIV-1 transmission: a prospective cohort study, The Lancet Infectious Diseases, (12), 19-26, (2011).

[29] Hethcote H.W., The mathematics of infectious diseases, SIAM Review 42(4), 599-653, (2000).

[30] Hethcote H.W. and Van Ark W. J., Epidemiological models for heterogeneous populations: Proportional mixing, parameter estimation and immunisation programs, Mathematical Biosciences, 84:85118, (1987).

[31] Hove-Musekwa S. D., Nyabadza F. and Mambili-Mamboundou H., Modelling hospitalization, homebased care, and individual withdrawal for people living with HIV/AIDS in high prevalence settings, British Medical Bulletin, (2011).

[32] Hickson R. I., Mercer G. N. and Lokuge K. M., Sensitivity analysis of a model for tubercolosis, 19th International Congress on Modelling and Simulation, (2011), http://mssanz.org.au/modsim2011, (accessed 01-05-2012).

[33] Hyman J. M. and Stanley J. Li, Disease transmission models with biased partnership selection, Applied Numerical Mathematics, (24), 379-392, (1997). 
[34] Hyman J. M. and Li J., Behavior changes in SIS STD models with selective mixing, Journal of Applied Mathematics, 57(4),1082-1094, (1997).

[35] IRIN,HIV/AIDS: Hormonal contraception advice not reaching women, (http://www.irinnews. org/printreport, (accessed 21-05-2012).

[36] Jacquez A. J., Simon C. P., Koopman J., Arbor A., Sattenspiel L. and Perry T., Modeling and analyzing HIV transmission: The effect of contact patterns, Mathematical Biosciences, 92, 119-199, (1988).

[37] Kapiga S. H. et al., The incidence of HIV infection among women using family planning methods in Dares Salaam, Tanzania, AIDS, 12(1), 7584, (1998).

[38] Kiddugavu M. et. al., Hormonal contraceptive use and HIV-1 infection in a population based cohort in Rakai, Uganda, AIDS, 17(2), 23340, (2003).

[39] Kleinschmidt I., et. al., Injectable progestin contraceptive use and risk of HIV infection in a South African family planning cohort, Contraception, 75(6), 4617, (2007).

[40] Kumwenda J. J., Makanani B., Taulo F., et al., Natural history and risk factors associated with early and established HIV type 1 infection among reproductive-age women in Malawi, Clinical Infectious Diseases, 46, 1913-1920, (2008).

[41] Lagarde E., Auvert B., Chege J. et al., The multicentre study of factors determining the different prevalences of HIV in sub-Saharan Africa, Condom use and its association with HIV/sexually transmitted diseases in four urban communities of sub-Saharan Africa, AIDS, 15, S71-S78, (2001).

[42] Lakshmikantham V., Leela S. and Martynyuk A. A., Stability analysis of nonlinear systems, Marcel Dekker, Inc., New York and Basel, 1-315, (1989).

[43] Lamptey P., Wigley M., Carr D. and Collymore Y., Facing the HIV/AIDS pandemic, Population Bulletin., 57(3),(2002).

[44] LaSalle J. P., The stability of dynamical systems, CBMS-NSF Regional Conference Series in Applied Mathematics, (25), (1976).

[45] Lavreys L. et. al., Hormonal contraception and risk of HIV-1 acquisition: results of a 10-year prospective study, AIDS, 18(4), 695697, 2004.

[46] Malunguza N., Mushayabasa S., Chiyaka C. and Mukandavire Z., Modelling the effects of condom use and antiretroviral therapy in controlling HIV/AIDS among heterosexuals, homosexuals and bisexuals, Computational and Mathematical Methods in Medicine, 11(3), 201-222, (2010).

[47] Malunguza N. J., Hove-Musekwa S. D., Musuka G. and Mukandavire Z., Investigating alcohol consumption as a risk factor for HIV transmission in heterosexual settings in sub-Saharan African communities., Bulletin of Mathematical Biology, 74(9), 2094-2124, (2012).

[48] McKay M. D., Conover W. J. and Beckman R. J., A comparison of three methods for selecting values of input variables in the analysis of output from a computer code , Technometrics, (21), 239-245, (1979).

[49] Marseillea E., Kahnb J. G., Billinghurst K. and Sabad J., Cost-effectiveness of the female condom in preventing HIV and STDs in commercial sex workers in rural South Africa, Social Science and Medicine, (52), 135-148, (2001). 
[50] Marx, P. A. et al., Progesterone implants enhance SIV vaginal transmission and early virus load, Nature Medicine, (2), 1084-1089, (1996).

[51] McCoy S. I., Zheng W., Montgomery E., Blanchard K., van Der Straten A., de Bruyn G., Padian N. S.,Oral and injectable contraception use and risk of HIV acquisition among women in the methods for improving reproductive health in Africa (MIRA) study, AIDS, (2012).

[52] Miller L., Patton D. L., Meier A., et al. Depomedroxyprogesterone-induced hypoestrogenism and changes in vaginal flora and epithelium, Journal of Obstetrics and Gynaecology, (96), 431-439, (2000).

[53] Moghadas S. M, Gumel A. B., McLeod R. G. and Gordon R., Could condoms stop the AIDS epidemic, Journal of Theoretical Medicine, (5), 171-181, (2003).

[54] Morrison C. S. and Cates W., Contraception, contraceptive technology, and STDs, In Holmes K, Stamm W, Wasserheit J and Cohen M (eds.), Sexually Transmitted Diseases 4th edn, 1493-1510, (2008).

[55] Morrison C. S., Skoler-Karpoff S., Kwok C., Chen P. L., van de Wijgert J., Gehret-Plagianos M., Patel S., Ahmed K., Ramjee G., Friedland B., Lahteenmaki P., Hormonal contraception and the risk of HIV acquisition among women in South AfricaAIDS, 26(4), 497-504, (2012).

[56] Morrison C. S. et. al., Hormonal contraception and HIV acquisition: reanalysis using marginal structural modeling, AIDS, 24(11), 1778-81, (2010).

[57] Morrison C. S., Skoler-Karpoff S., Kwok C., Chen P. L., van de Wijgert J., Gehret-Plagianos M., Patel S., Ahmed K., Ramjee G., Friedland B. and Lahteenmaki P., Hormonal contraception and the risk of HIV acquisition among women in South Africa, AIDS, 26(4), 497-504, (2012).

[58] Mukandavire Z. and Garira W., HIV/AIDS model for assessing the effects of prophylactic sterilizing vaccines, condoms and treatment with amelioration, Journal of Biological Systems, 14(3), 323-355, (2006).

[59] Mukandavire Z., Chiyaka C., Magombedze G., Musuka G. and Malunguza N., Assessing the effects of homosexuals and bisexuals on the intrinsic dynamics of HIV/AIDS in African heterosexual settings, Mathematical Computational Modelling, 49(9-10), 1869-1882, (2009).

[60] Mukandavire Z. and Garira W., Sex-structured HIV/AIDS model to analyse the effects of condom use with application to Zimbabwe, Journal of Mathematical Biology, 54(5), 669-699, (2007).

[61] Mukandavire Z., Malunguza N. J., Chiyaka C., Musuka G. and Tchuenche J. M., HIV/AIDS model assessing the effects of gender-inequality affecting women in African heterosexual settings, International Journal of Biomathematics, 3(1), 43-67, (2009).

[62] Myer L., et al., Prospective study of hormonal contraception and women's risk of HIV infection in South Africa, International Journal of Epidemiology, 36(1), 166-74, (2007).

[63] Nold A., Heterogeneity in disease-transmission modeling, Mathematical Biosciences, 5, 227-240, (1980).

[64] Over M. and Piot P., HIV infection and sexually transmitted diseases, In D. T. Jamison, et al. (Ed.), Disease Control Priorities in Developing Countries, 455-527, (1993).

[65] Polis C. B. and Curtis K. M., Use of hormonal contraceptives and HIV acquisition in women: a systematic review of the epidemiological evidence, Lancet Infectious Diseases, 13(9), 797-808, (2013). 
[66] Polis C. B., Phillips S. J. and Curtis K. M., Hormonal contraceptive use and female-to-male HIV transmission: a systematic review of the epidemiologic evidence, AIDS, 27(4), 493-505, (2013).

[67] Polis C. B., et. al., Hormonal contraceptive methods and risk of HIV acquisition in women: a systematic review of epidemiological evidence, Contraception, 90(4), 360-90, (2014).

[68] Nyabadza F., Mukandavire Z. and Hove-Musekwa S. D., Modelling the HIV/AIDS epidemic trends in South Africa: Insights from a simple mathematical model, Nonlinear Analysis: Theory, Methods and Applications, 12(4), 2091-2104, (2011).

[69] Okech T. C., Wawire N. W. and Mburu T. K., Contraceptive use among women of reproductive age in Kenya's city slums, International Journal of Business and Social Science, 2(1), (2011).

[70] Ramjee G. and Wand H., Population-level impact of hormonal contraception on incidence of HIV infection and pregnancy in women in Durban, South Africa, Bulletin of World Health Organisation, (90), 748-755, (2012).

[71] Reid S. E. et. al., Pregnancy, contraceptive use, and HIV acquisition in HPTN 039: relevance for HIV prevention trials among African women, Journal of Acquired Immune Deficiency Syndromes, 53(5), 606-13, (2010).

[72] Shanti R. C., Sub-Saharan Africa at the turning point- contraceptive use gains acceptance, http:/findarticles.com/p/articles/,(accessed 21-05-2012).

[73] Singh S., Sedgh G. and Hussain R. 2010, Unintended pregnancy: worldwide levels, trends, and outcomes, Studies in Familly Planning, 41(4), 241-50, (2010).

[74] Tanser F., Bärnighausen T., Hund L., Garnett G. P., McGrath N. and Newell M., Effect of concurrent sexual partnerships on rate of new HIV infections in a high-prevalence, rural South African population: a cohort study The Lancet, 378(9787), 247-255, (2011).

[75] Thomas G. and Lungu E., M., The influence of heavy alcohol consumption on HIV Infection and progression, Journal of Biological Systems, 17(4), 685-712, (2009).

[76] UNAIDS fact sheets, (www.unaids.org), (accessed 31-08-2012).

[77] UNAIDS 1998, Report on the global HIV/AIDS epidemic, http://data.unaids.org, (accessed 25-012015).

[78] UNAIDS 2000, Report on the global HIV/AIDS epidemic, http://data.unaids.org, (accessed 0\%-022012).

[79] UNAIDS 2001, We can end poverty by 2015, http://www.un.org/millenniumgoals/bkgd.shtml, (accessed 15-03-2014).

[80] UNAIDS 2005, Evidence for HIV decline in Zimbabwe: A comprehensive review of the epidemiological data, (2005)

http://data.unaids.org/Publications/IRC-pub06/zimbabwe_epi_report_nov05_en.pdf

[81] UNAIDS 2006, Report on the Global HIV/AIDS Epidemic, Joint United Nations Programme on HIV/AIDS, http://www.unaids.org, (accessed 25-11-2011).

[82] UNAIDS 2008, Report on the global AIDS epidemic, http://www.unaids.org, (accessed 08-08-2014). 
[83] UNGASS (2010) Zimbabwe UNGASS Country Progress Report, http:data. unaids .org/Report/zimbabwe-2008-country-progress, (accessed 31-08-2012).

[84] UNAIDS 2010, Global report: UNAIDS report on the global AIDS epidemic, www.unaids.org, (accessed 24-07-2013), (2010).

[85] van den Driessche P. and Watmough J., Reproduction numbers and sub-threshold endemic equilibria for compartmental models of disease transmission, John A. Jacquez memorial volume, Mathematical Biosciences, (180), 29-48, (2002).

[86] Wadman M., Contraceptive risk of HIV long suspected, Nature, http://www. nature.com/news/2011/111007/full/news.2011.582.html, (accessed 01-11-2012)

[87] Wand H. and Ramjee G., The effects of injectable hormonal contraceptives on HIV seroconversion and on sexually transmitted infections, AIDS, 26(3), 375-80, (2012).

[88] Wang C. C., Kreiss J. K. and Reilly, Risk of HIV infection in oral contraceptive pill users: a metaanalysis, Journal of Acquired Immune Deficiency Syndromes, (21), 51-58, (1999).

[89] World Development Indicators. Washington, DC: World Bank, http://www. workinfo.com/Workforce/20997-devindicators.pdf, (accessed 12-12-2012).

[90] Yeatman S. E. and Trinitapoli J., Beyond Denomination: The Relationship between Religion and Family Planning in Rural Malawi, PMC, 9(55), 1851-1882, (2010).

[91] ZIMDATA (2011), http://www.zimstat.co.zw/index.php?, (accessed 25-11-2012)

\section{Appendix A}

Substituting the values of $S_{f_{c}}^{*}, S_{f_{n}}^{*}, S_{f_{h}}^{*}, S_{m}^{*}, I_{f_{c}}^{*}, I_{f_{n}}^{*}, I_{f_{h}}^{*}, I_{m}^{*}$ into $\lambda_{f_{c}}^{*}, \lambda_{f_{n}}^{*}, \lambda_{f_{h}}^{*}$ and $\lambda_{m}^{*}$ gives

$$
\left\{\begin{array}{l}
\lambda_{f_{n}}^{*}=\frac{c_{f} \beta_{m} \lambda_{m}^{*} \rho_{f_{n} m}}{\left(\mathcal{K}_{1}+\lambda_{m}^{*}\right)} \\
\lambda_{f_{c}}^{*}=\frac{\left(1-p_{c}\right) c_{f} \beta_{m} \lambda_{m}^{*} \rho_{f_{c} m}}{\left(\mathcal{K}_{1}+\lambda_{m}^{*}\right)} \\
\lambda_{f_{h}}^{*}=\frac{\chi c_{f} \beta_{m} \lambda_{m}^{*} \rho_{f_{h} m}}{\left(\mathcal{K}_{1}+\lambda_{m}^{*}\right)}
\end{array}\right.
$$

Using the relationship $\lambda_{f_{c}}^{*}=\frac{\left(1-p_{c}\right) \rho_{f m_{c}}}{\rho_{f m_{n}}} \lambda_{f_{n}}^{*}, \lambda_{f_{h}}^{*}=\frac{\chi \rho_{f m_{h}}}{\rho_{f_{n} m}} \lambda_{f_{n}}^{*}$ and $\lambda_{f_{n}}^{*}=\frac{c_{f} \beta_{m} \lambda_{m}^{*} \rho_{f_{n} m}}{\left(\mathcal{K}_{1}+\lambda_{m}^{*}\right)}$ and solving for $\lambda_{m}^{*}$ where

$$
\left\{\begin{array}{l}
\mathcal{A}_{1}=\mathcal{K}_{3} \mathcal{K}_{5} \mathcal{K}_{8} \delta_{2}+\mathcal{K}_{4}\left(\mathcal{K}_{5} \mathcal{K}_{6} \delta_{1}+\mathcal{K}_{3} \mathcal{K}_{7} \delta_{3}\right) \\
\mathcal{A}_{2}=\mathcal{K}_{1}\left(\mathcal{K}_{2}\left(\mathcal{K}_{4} \mathcal{K}_{6} \delta_{1}+\mathcal{K}_{5} \mathcal{K}_{6} \delta_{1}+\mathcal{K}_{3} \mathcal{K}_{8} \delta_{2}+\mathcal{K}_{5} \mathcal{K}_{8} \delta_{2}+\left(\mathcal{K}_{3}+\mathcal{K}_{4}\right) \mathcal{K}_{7} \delta_{3}\right)\right. \\
\left.+\mathcal{K}_{1}\left(\mathcal{K}_{4} \mathcal{K}_{5}\left(\mathcal{R}_{h}-\mathcal{R}_{H}^{2}+\mathcal{R}_{n}+\delta_{1}\right)+\mathcal{K}_{3}\left(\mathcal{K}_{5}\left(\mathcal{R}_{c}+\mathcal{R}_{h}-\mathcal{R}_{H}^{2}+\delta_{2}\right)+\mathcal{K}_{4}\left(\mathcal{R}_{c}-\mathcal{R}_{H}^{2}+\mathcal{R}_{n}+\delta_{3}\right)\right)\right)\right) \\
\mathcal{A}_{3}=\mathcal{K}_{1}^{2} \mathcal{K}_{2}\left(\mathcal { K } _ { 1 } \left(\mathcal{K}_{3}\left(1+\mathcal{R}_{c}-\mathcal{R}_{H}^{2}-\delta_{1}\right)-\mathcal{K}_{4}\left(-1+\mathcal{R}_{H}^{2}-\mathcal{R}_{n}+\delta_{2}\right)+\mathcal{K}_{5}\left(1+\mathcal{R}_{h}-\mathcal{R}_{H}^{2}-\delta_{3}\right)\right.\right. \\
\left.\left.+\mathcal{K}_{2}\left(\mathcal{K}_{6} \delta_{1}+\mathcal{K}_{8} \delta_{2}+\mathcal{K}_{7} \delta_{3}\right)\right)\right) \\
\mathcal{A}_{3}=\mathcal{K}_{1}^{4} \mathcal{K}_{2}^{2}\left(\mathcal{R}_{H}^{2}-1\right)
\end{array}\right.
$$


and

$$
\left\{\begin{array}{l}
\mathcal{K}_{3}=\left(\mathcal{K}_{2}+\left(1-p_{c}\right) c_{f} \beta_{m} \rho_{f_{c} m}\right), \mathcal{K}_{4}=\left(\mathcal{K}_{2}+c_{f} \beta_{m} \rho_{f_{n} m}\right), \mathcal{K}_{5}=\left(\mathcal{K}_{2}+\chi c_{f} \beta_{m} \rho_{f_{h} m}\right), \\
\mathcal{K}_{6}=\left(\mathcal{K}_{1}+\left(1-p_{c}\right) c_{f} \beta_{m} \rho_{f_{c} m}\right), \mathcal{K}_{7}=\left(\mathcal{K}_{1}+\chi c_{f} \beta_{m} \rho_{f_{h} m}\right), \mathcal{K}_{8}=\left(\mathcal{K}_{1}+c_{f} \beta_{m} \rho_{f_{n} m}\right), \\
\mathcal{R}_{n}=\frac{\delta_{2} c_{f} \beta_{m} \rho_{f_{n} m} c_{m} \beta_{f}}{\mathcal{K}_{1}^{2}}, \mathcal{R}_{c}=\frac{\delta_{1}\left(-1+p_{c}\right)^{2} c_{f} c_{m} \beta_{f} \beta_{m} \rho_{f_{c} m}^{2}}{\mathcal{K}_{1}^{2}}, \mathcal{R}_{h}=\frac{\delta_{3} \theta \chi c_{f} c_{m} \beta_{f} \beta_{m} \rho_{f_{h} m}^{2}}{\mathcal{K}_{1}^{2}}
\end{array}\right.
$$

Further algebraic manipulations result in the following quatic function shown in equation (17) whose solution is shown in equations (18),(19),(20) and (21).

\section{Appendix B}

Global stability of endemic equilibrium.

Proof. At equilibrium, the following relations hold

$$
\left.\begin{array}{l}
\delta_{1} \phi \Lambda=\lambda_{f_{c}}^{*} S_{f_{c}}^{*}+\mathcal{K}_{2} S_{f_{c}}^{*}, \\
\delta_{2} \phi \Lambda=\lambda_{f_{n}}^{*} S_{f_{n}}^{*}+\mathcal{K}_{2} S_{f_{n}}^{*}, \\
\delta_{3} \phi \Lambda=\lambda_{f_{h}}^{*} S_{f_{h}}^{*}+\mathcal{K}_{2} S_{f_{h}}^{*}, \\
(1-\phi) \Lambda=\lambda_{m}^{*} S_{m}^{*}+\mathcal{K}_{2} S_{m}^{*},
\end{array}\right\}
$$

and

$$
\left\{\begin{array}{l}
\mathcal{K}_{1} I_{f_{c}}^{*}=\lambda_{f_{c}}^{*} S_{f_{c}}^{*}, \mathcal{K}_{1} I_{f_{n}}^{*}=\lambda_{f_{n}}^{*} S_{f_{n}}^{*} \\
\mathcal{K}_{1} I_{f_{h}}^{*}=\lambda_{f_{h}}^{*} S_{f_{h}}^{*}, \mathcal{K}_{1} I_{m}^{*}=\lambda_{m}^{*} S_{m}^{*}
\end{array}\right.
$$

Consider the candidate Lyapunov function $V$, such that,

$$
\begin{aligned}
V & =S_{f_{c}}-S_{f_{c}}^{*}-S_{f_{c}}^{*} \ln \frac{S_{f_{c}}}{S_{f_{c}}^{*}}+S_{f_{n}}-S_{f_{n}}^{*}-S_{f_{n}}^{*} \ln \frac{S_{f_{n}}}{S_{f_{n}}^{*}}+S_{f_{h}}-S_{f_{h}}^{*}-S_{f_{h}}^{*} \ln \frac{S_{f_{h}}}{S_{f_{h}}^{*}}+S_{m}-S_{m}^{*}-S_{m}^{*} \ln \frac{S_{m}}{S_{m}^{*}} \\
& +I_{f_{c}}-I_{f_{c}}^{*}-I_{f_{c}}^{*} \ln \frac{I_{f_{c}}}{I_{f_{c}}^{*}}+I_{f_{n}}-I_{f_{n}}^{*}-I_{f_{n}}^{*} \ln \frac{I_{f_{n}}}{I_{f_{n}}^{*}}+I_{f_{h}}-I_{f_{h}}^{*}-I_{f_{h}}^{*} \ln \frac{I_{f_{h}}}{I_{f_{h}}^{*}}+I_{m}-I_{m}^{*}-I_{m}^{*} \ln \frac{I_{m}}{I_{m}^{*}} .
\end{aligned}
$$

Clearly $S_{f_{c}}-S_{f_{c}}^{*}$ is an increasing polynomial and $S_{f_{c}}^{*} \ln \frac{S_{f_{c}}}{S_{f_{c}}^{*}}$ is logarithmic function and using the same argument on the rest of the variables the polynomial will outgrow the logarithmic function and therefore

$$
\left\{\begin{array}{l}
V\left(S_{f_{c}}, S_{f_{n}}, S_{f_{h}}, S_{m}, I_{f_{c}}, I_{f_{n}}, I_{f_{h}}, I_{m}\right)>0, S_{f_{c}}>S_{f_{c}}^{*}, S_{f_{n}}>S_{f_{n}}^{*}, S_{f_{h}}>S_{f_{h}}^{*}, S_{m}>S_{m}^{*}, I_{f_{c}}>I_{f_{c}}^{*}, \\
I_{f_{n}}>I_{f_{n}}^{*}, I_{f_{h}} I_{f_{h}}^{*}, I_{m}>I_{m}^{*} \\
V\left(S_{f_{c}}^{*}, S_{f_{n}}^{*}, S_{f_{h}}^{*}, S_{m}^{*}, I_{f_{c}}^{*}, I_{f_{n}}^{*}, I_{f_{h}}^{*}, I_{m}^{*}\right)=0
\end{array}\right.
$$

What is left is to prove that

$$
\dot{V}<0
$$

The time derivative of $V$ along the solution path is given by

$$
\begin{aligned}
\dot{V} & =\dot{S_{f_{c}}}\left[1-\frac{S_{f_{c}}^{*}}{S_{f_{c}}}\right]+\dot{S_{f_{n}}}\left[1-\frac{S_{f_{n}}^{*}}{S_{f_{n}}}\right]+\dot{S_{f_{h}}}\left[1-\frac{S_{f_{h}}^{*}}{S_{f_{h}}}\right]+\dot{S_{m}}\left[1-\frac{S_{m}^{*}}{S_{m}}\right]+\dot{I_{f_{c}}}\left[1-\frac{I_{f_{c}}^{*}}{I_{f_{c}}}\right] \\
& +\dot{I_{f_{n}}}\left[1-\frac{I_{f_{n}}^{*}}{I_{f_{n}}}\right]+\dot{I_{f_{h}}}\left[1-\frac{I_{f_{h}}^{*}}{I_{f_{h}}}\right]+\dot{I_{m}}\left[1-\frac{I_{m}^{*}}{I_{m}}\right]
\end{aligned}
$$


Substituting equilibrium values and re-writing expressions for the derivatives of the state variables we obtain the following system

$$
\begin{aligned}
& \dot{S_{f_{c}}}=\lambda_{f_{c}}^{*} S_{f_{c}}^{*}+\mathcal{K}_{2} S_{f_{c}}^{*}-\lambda_{f_{c}}^{*} S_{f_{c}}^{*} \frac{\lambda_{f_{c}} S_{f_{c}}}{\lambda_{f_{c}}^{*} S_{f_{c}}^{*}}-\mathcal{K}_{2} S_{f_{c}}^{*} \frac{S_{f_{c}}}{S_{f_{c}}^{*}}, \\
& \dot{I_{f_{c}}}=\lambda_{f_{c}}^{*} S_{f_{c}}^{*} \frac{\lambda_{f_{c}} S_{f_{c}}}{\lambda_{f_{c}}^{*} S_{f_{c}}^{*}}-\mathcal{K}_{1} I_{f_{c}}^{*} \frac{I_{f_{c}}}{I_{f_{c}}^{*}}, \\
& \dot{S_{f_{n}}}=\lambda_{f_{n}}^{*} S_{f_{n}}^{*}+\mathcal{K}_{2} S_{f_{n}}^{*}-\lambda_{f_{n}}^{*} S_{f_{n}}^{*} \frac{\lambda_{f_{n}} S_{f_{n}}}{\lambda_{f_{n}}^{*} S_{f_{n}}^{*}}-\mathcal{K}_{2} S_{f_{n}}^{*} \frac{S_{f_{n}}}{S_{f_{n}}^{*}}, \\
& \dot{I_{f_{n}}}=\lambda_{f_{n}}^{*} S_{f_{n}}^{*} \frac{\lambda_{f_{n}} S_{f_{n}}}{\lambda_{f_{n}}^{*} S_{f_{n}}^{*}}-\mathcal{K}_{1} I_{f_{n}}^{*} \frac{I_{f_{n}}}{I_{f_{n}}^{*}}, \\
& \dot{S_{f_{h}}}=\lambda_{f_{h}}^{*} S_{f_{h}}^{*}+\mathcal{K}_{2} S_{f_{h}}^{*}-\lambda_{f_{h}}^{*} S_{f_{h}}^{*} \frac{\lambda_{f_{h}} S_{f_{h}}}{\lambda_{f_{h}}^{*} S_{f_{h}}^{*}}-\mathcal{K}_{2} S_{f_{h}}^{*} \frac{S_{f_{h}}}{S_{f_{h}}^{*}}, \\
& \dot{I_{f_{h}}}=\lambda_{f_{h}}^{*} S_{f_{h}}^{*} \frac{\lambda_{f_{h}} S_{f_{h}}}{\lambda_{f_{h}}^{*} S_{f_{h}}^{*}}-\mathcal{K}_{1} I_{f_{h}}^{*} \frac{I_{f_{h}}}{I_{f_{h}}^{*}}, \\
& \dot{S_{m}}=\lambda_{m}^{*} S_{m}^{*}+\mathcal{K}_{2} S_{m}^{*}-\lambda_{m}^{*} S_{m}^{*} \frac{\lambda_{m} S_{m}}{\lambda_{m}^{*} S_{m}^{*}}-\mathcal{K}_{2} S_{m}^{*} \frac{S_{m}}{S_{m}^{*}}, \\
& \dot{I_{m}}=\lambda_{m}^{*} S_{m}^{*} \frac{\lambda_{m} S_{m}}{\lambda_{m}^{*} S_{m}^{*}}-\mathcal{K}_{1} I_{m}^{*} \frac{I_{m}}{I_{m}^{*}} .
\end{aligned}
$$

Substituting into equation (B.2), the re-arranged expressions for the derivatives of the state variables as well as the equilibrium values of $\mathcal{K}_{1} I_{f_{c}}^{*}, \mathcal{K}_{1} I_{f_{n}}^{*}, \mathcal{K}_{1} I_{f_{h}}^{*}$ and $\mathcal{K}_{1} I_{m}^{*}$, we obtain the following expression,

$$
\begin{aligned}
& \left(\lambda_{f_{c}}^{*} S_{f_{c}}^{*}+\mathcal{K}_{2} S_{f_{c}}^{*}-\lambda_{f_{c}}^{*} S_{f_{c}}^{*} \frac{\lambda_{f_{c}} S_{f_{c}}}{\lambda_{f_{c}}^{*} S_{f_{c}}^{*}}-\mathcal{K}_{2} S_{f_{c}}^{*} \frac{S_{f_{c}}}{S_{f_{c}}^{*}}-\lambda_{f_{c}}^{*} S_{f_{c}}^{*} \frac{S_{f_{c}}^{*}}{S_{f_{c}}}-\mathcal{K}_{2} S_{f_{c}}^{*} \frac{S_{f_{c}}^{*}}{S_{f_{c}}}+\lambda_{f_{c}}^{*} S_{f_{c}}^{*} \frac{\lambda_{f_{c}}}{\lambda_{f_{c}}^{*}}+\mathcal{K}_{2} S_{f_{c}}^{*}\right. \\
& +\lambda_{f_{c}}^{*} S_{f_{c}}^{*} \frac{\lambda_{f_{c}} S_{f_{c}}}{\lambda_{f_{c}}^{*} S_{f_{c}}^{*}}-\lambda_{f_{c}}^{*} S_{f_{c}}^{*} \frac{I_{f_{c}}}{I_{f_{c}}^{*}}-\lambda_{f_{c}}^{*} S_{f_{c}}^{*} \frac{\lambda_{f_{c}} S_{f_{c}} I_{f_{c}}^{*}}{\lambda_{f_{c}}^{*} S_{f_{c}}^{*} I_{f_{c}}}+\lambda_{f_{c}}^{*} S_{f_{c}}^{*} \\
& +\lambda_{f_{n}}^{*} S_{f_{n}}^{*}+\mathcal{K}_{2} S_{f_{n}}^{*}-\lambda_{f_{n}}^{*} S_{f_{n}}^{*} \frac{\lambda_{f_{n}} S_{f_{n}}}{\lambda_{f_{n}}^{*} S_{f_{n}}^{*}}-\mathcal{K}_{2} S_{f_{n}}^{*} \frac{S_{f_{n}}}{S_{f_{n}}^{*}}-\lambda_{f_{n}}^{*} S_{f_{n}}^{*} \frac{S_{f_{n}}^{*}}{S_{f_{n}}}-\mathcal{K}_{2} S_{f_{n}}^{*} \frac{S_{f_{n}}^{*}}{S_{f_{n}}}+\lambda_{f_{n}}^{*} S_{f_{n}}^{*} \frac{\lambda_{f_{n}}}{\lambda_{f_{n}}^{*}}+\mathcal{K}_{2} S_{f_{n}}^{*} \\
& \dot{V}=\left\{+\lambda_{f_{n}}^{*} S_{f_{n}}^{*} \frac{\lambda_{f_{n}} S_{f_{n}}}{\lambda_{f_{n}}^{*} S_{f_{n}}^{*}}-\lambda_{f_{n}}^{*} S_{f_{n}}^{*} \frac{I_{f_{n}}}{I_{f_{n}}^{*}}-\lambda_{f_{n}}^{*} S_{f_{n}}^{*} \frac{\lambda_{f_{n}} S_{f_{n}} I_{f_{n}}^{*}}{\lambda_{f_{n}}^{*} S_{f_{n}}^{*} I_{f_{n}}}+\lambda_{f_{n}}^{*} S_{f_{n}}^{*}\right. \\
& +\lambda_{f_{h}}^{*} S_{f_{h}}^{*}+\mathcal{K}_{2} S_{f_{h}}^{*}-\lambda_{f_{h}}^{*} S_{f_{h}}^{*} \frac{\lambda_{f_{h}} S_{f_{h}}}{\lambda_{f_{h}}^{*} S_{f_{h}}^{*}}-\mathcal{K}_{2} S_{f_{h}}^{*} \frac{S_{f_{h}}}{S_{f_{h}}^{*}}-\lambda_{f_{h}}^{*} S_{f_{h}}^{*} \frac{S_{f_{h}}^{*}}{S_{f_{h}}}-\mathcal{K}_{2} S_{f_{h}}^{*} \frac{S_{f_{h}}^{*}}{S_{f_{h}}}+\lambda_{f_{h}}^{*} S_{f_{h}}^{*} \frac{\lambda_{f_{h}}}{\lambda_{f_{h}}^{*}}+\mathcal{K}_{2} S_{f_{h}}^{*} \\
& +\lambda_{f_{h}}^{*} S_{f_{h}}^{*} \frac{\lambda_{f_{h}} S_{f_{h}}}{\lambda_{f_{h}}^{*} S_{f_{h}}^{*}}-\lambda_{f_{h}}^{*} S_{f_{h}}^{*} \frac{I_{f_{h}}}{I_{f_{h}}^{*}}-\lambda_{f_{h}}^{*} S_{f_{h}}^{*} \frac{\lambda_{f_{h}} S_{f_{h}} I_{f_{h}}^{*}}{\lambda_{f_{h}}^{*} S_{f_{h}}^{*} I_{f_{h}}}+\lambda_{f_{h}}^{*} S_{f_{h}}^{*} \\
& +\lambda_{m}^{*} S_{m}^{*}+\mathcal{K}_{2} S_{m}^{*}-\lambda_{m}^{*} S_{m}^{*} \frac{\lambda_{m} S_{m}}{\lambda_{m}^{*} S_{m}^{*}}-\mathcal{K}_{2} S_{m}^{*} \frac{S_{m}}{S_{m}^{*}}-\lambda_{m}^{*} S_{m}^{*} \frac{S_{m}^{*}}{S_{m}}-\mathcal{K}_{2} S_{m}^{*} \frac{S_{m}^{*}}{S_{m}}+\lambda_{m}^{*} S_{m}^{*} \frac{\lambda_{m}}{\lambda_{m}^{*}}+\mathcal{K}_{2} S_{m}^{*} \\
& +\lambda_{m}^{*} S_{m}^{*} \frac{\lambda_{m} S_{m}}{\lambda_{m}^{*} S_{m}^{*}}-\lambda_{m}^{*} S_{m}^{*} \frac{I_{m}}{I_{m}^{*}}-\lambda_{m}^{*} S_{m}^{*} \frac{\lambda_{m} S_{m} I_{m}^{*}}{\lambda_{m}^{*} S_{m}^{*} I_{m}}+\lambda_{m}^{*} S_{m}^{*} .
\end{aligned}
$$

Collecting like terms, the derivative reduces to

$$
\dot{V}=\left\{\begin{array}{l}
\mathcal{K}_{2} S_{f_{c}}^{*}\left[2-\frac{S_{f_{c}}}{S_{f_{c}}^{*}}-\frac{S_{f_{c}}^{*}}{S_{f_{c}}}\right]+\lambda_{f_{c}}^{*} S_{f_{c}}^{*}\left[1-\frac{I_{f_{c}}}{I_{f_{c}}^{*}}-\frac{S_{f_{c}}^{*}}{S_{f_{c}}}\right]+\lambda_{f_{c}}^{*} S_{f_{c}}^{*} \frac{\lambda_{f_{c}}}{\lambda_{f_{c}}^{*}}\left[1-\frac{S_{f_{c}} I_{f_{c}}^{*}}{S_{f_{c}}^{*} I_{f_{c}}}\right] \\
+\mathcal{K}_{2} S_{f_{n}}^{*}\left[2-\frac{S_{f_{n}}}{S_{f_{n}}^{*}}-\frac{S_{f_{n}}^{*}}{S_{f_{n}}}\right]+\lambda_{f_{n}}^{*} S_{f_{n}}^{*}\left[1-\frac{I_{f_{n}}}{I_{f_{n}}^{*}}-\frac{S_{f_{n}}^{*}}{S_{f_{n}}}\right]+\lambda_{f_{n}}^{*} S_{f_{n}}^{*} \frac{\lambda_{f_{n}}}{\lambda_{f_{n}}^{*}}\left[1-\frac{S_{f_{n}}}{S_{f_{n}}^{*} I_{f_{n}}}\right] \\
+\mathcal{K}_{2} S_{f_{h}}^{*}\left[2-\frac{S_{f_{h}}}{S_{f_{h}}^{*}}-\frac{S_{f_{h}}^{*}}{S_{f_{h}}}\right]+\lambda_{f_{h}}^{*} S_{f_{h}}^{*}\left[1-\frac{I_{f_{h}}}{I_{f_{h}}^{*}}-\frac{S_{f_{h}}^{*}}{S_{f_{h}}}\right]+\lambda_{f_{h}}^{*} S_{f_{h}}^{*} \frac{\lambda_{f_{h}}}{\lambda_{f_{h}}^{*}}\left[1-\frac{S_{f_{h}}^{*}}{S_{f_{h}}^{*} I_{f_{h}}}\right] \\
+\mathcal{K}_{2} S_{m}^{*}\left[2-\frac{S_{m}}{S_{m}^{*}}-\frac{S_{m}^{*}}{S_{m}}\right]+\lambda_{m}^{*} S_{m}^{*}\left[1-\frac{I_{m}}{I_{m}^{*}}-\frac{S_{m}^{*}}{S_{m}}\right]+\lambda_{m}^{*} S_{m}^{*} \frac{\lambda_{m}}{\lambda_{m}^{*}}\left[1-\frac{S_{m} I_{m}^{*}}{S_{m}^{*} I_{m}}\right] .
\end{array}\right.
$$


Substituting equilibrium values in Theorem 4,

$$
\dot{V}=\left\{\begin{array}{l}
-\mathcal{K}_{2} S_{f_{c}}^{*} \frac{\left[S_{f_{c}}-S_{f_{c}}^{*}\right]^{2}}{S_{f_{c}}^{*} S_{f_{c}}}+\lambda_{f_{c}}^{*} S_{f_{c}}^{*}\left[1-\frac{I_{f_{c}}}{I_{f_{c}}^{*}}-\frac{S_{f_{c}}^{*}}{S_{f_{c}}}\right] \\
-\mathcal{K}_{2} S_{f_{n}}^{*} \frac{\left[S_{f_{n}}-S_{f_{n}}^{*}\right]^{2}}{S_{f_{n}}^{*} S_{f_{n}}}+\lambda_{f_{n}}^{*} S_{f_{n}}^{*}\left[1-\frac{I_{f_{n}}}{I_{f_{n}}^{*}}-\frac{S_{f_{n}}^{*}}{S_{f_{n}}}\right] \\
-\mathcal{K}_{2} S_{f_{h}}^{*} \frac{\left[S_{f_{h}}-S_{f_{h}}^{*}\right]^{2}}{S_{f_{h}}^{*} S_{f_{h}}}+\lambda_{f_{h}}^{*} S_{f_{h}}^{*}\left[1-\frac{I_{f_{h}}}{I_{f_{h}}^{*}}-\frac{S_{f_{h}}^{*}}{S_{f_{h}}}\right] \\
-\mathcal{K}_{2} S_{m}^{*} \frac{\left[S_{m}-S_{m}^{*}\right]^{2}}{S_{m}^{*} S_{m}}+\lambda_{m}^{*} S_{m}^{*}\left[1-\frac{I_{m}}{I_{m}^{*}}-\frac{S_{m}^{*}}{S_{m}}\right] .
\end{array}\right.
$$

Based on the conditions for the validity of the candidate Lyapunov function $S_{f_{c}}>S_{f_{c}}^{*}, S_{f_{n}}>$ $S_{f_{n}}^{*}, S_{f_{h}}>S_{f_{h}}^{*}, S_{m}>S_{m}^{*}, I_{f_{c}}>I_{f_{c}}^{*}, \dot{V} \leq 0$. We used the Lyapunov stability theorem to show that $\dot{V}<0$ for all

$$
\left(S_{f_{c}}^{*}, S_{f_{n}}^{*}, S_{f_{h}}^{*}, S_{m}^{*}, I_{f_{c}}^{*}, I_{f_{n}}^{*}, I_{f_{h}}^{*}, I_{m}^{*}\right)>0 \in \mathcal{D}
$$

and the strict equality $\dot{V}=0$ holds only for $S_{f_{c}}=S_{f_{c}}^{*}, S_{f_{n}}=S_{f_{n}}^{*}, S_{f_{h}}=S_{f_{h}}^{*}, S_{m}=S_{m}^{*}, I_{f_{c}}=$ $I_{f_{c}}^{*}, I_{f_{n}}=I_{f_{n}}^{*}, I_{f_{h}}=I_{f_{h}}^{*}$ and $I_{m}=I_{m}^{*}$. Then the only equilibrium state $\xi^{*}$ is the only positively invariant set of the endemic solution for model system (5) contained entirely in $\mathcal{D}$ and hence by the asymptotic stability theorem in [44], the endemic equilibrium state $\xi^{*}$ is a sink. This completes the proof. 


\section{Figures}

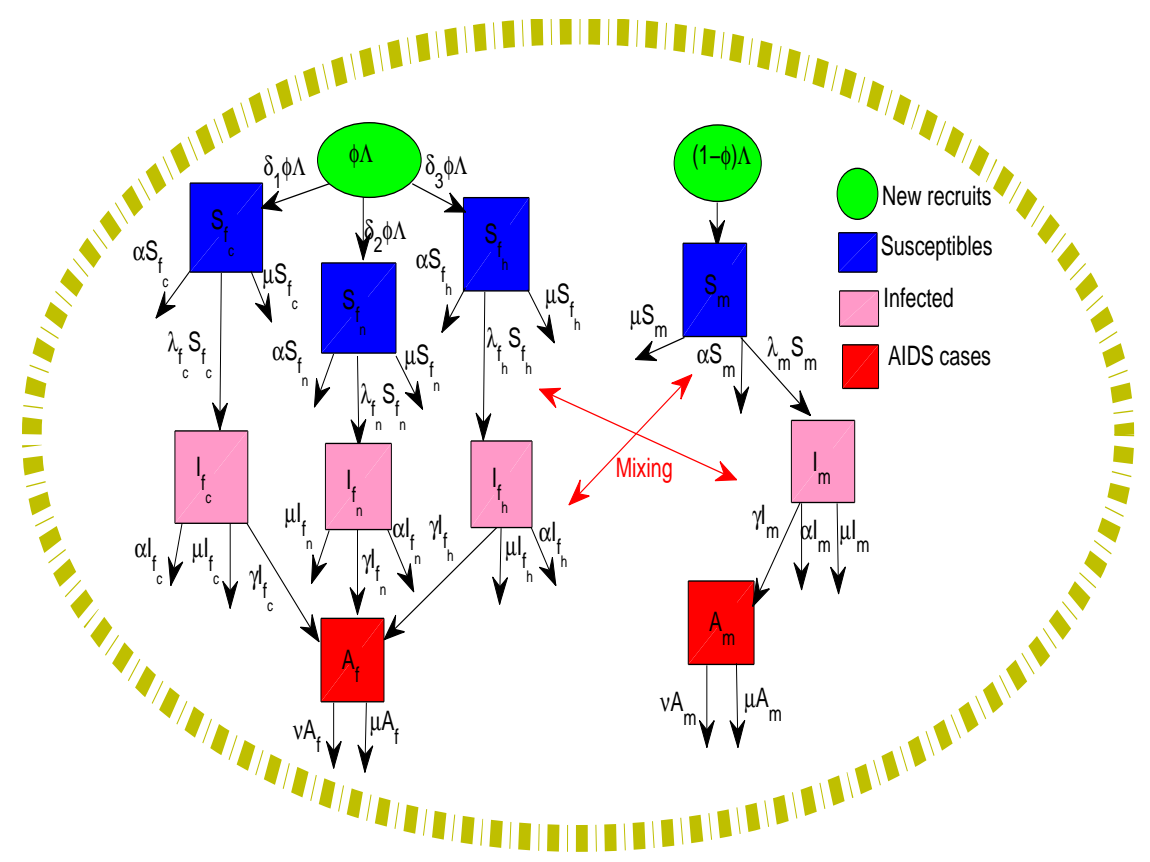

Figure 1: Model flow diagram showing the movement of people from Susceptible $S_{i}$ (blue in colour), Infected $I_{i}$ (pink in colour) to AIDS $A_{i}$ (red in colour) classes. The two compartments denote male and female with the red double arrows denoting heterogeneous sexual interaction.

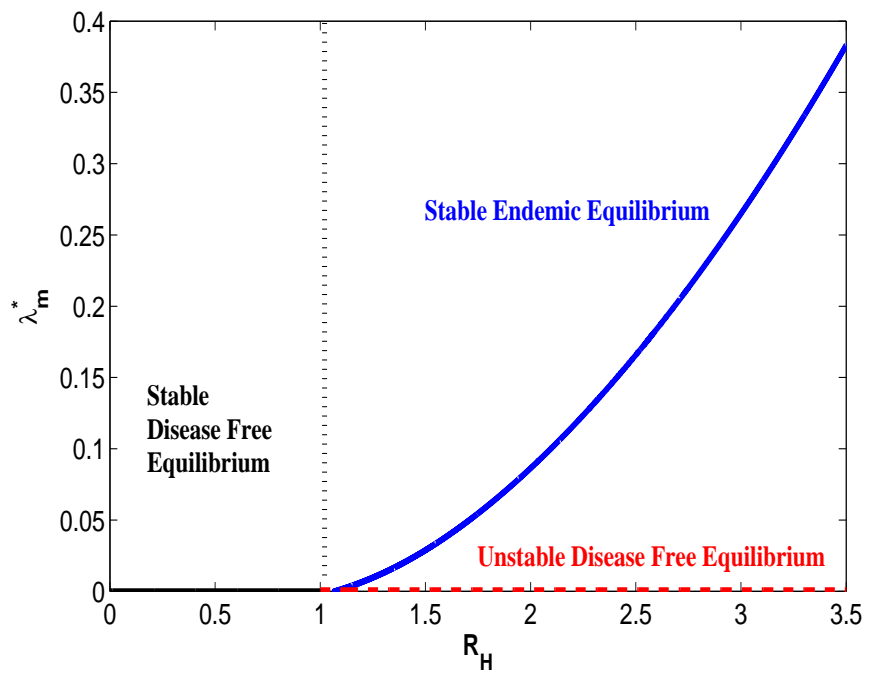

Figure 2: Plot of $\mathcal{R}_{H}$ against $\lambda_{m}^{*}$ for varying values of $\beta_{f}$ increasing from 0 to 1 in step sizes of 0.1 and all other parameters are as given in Table 2 . 

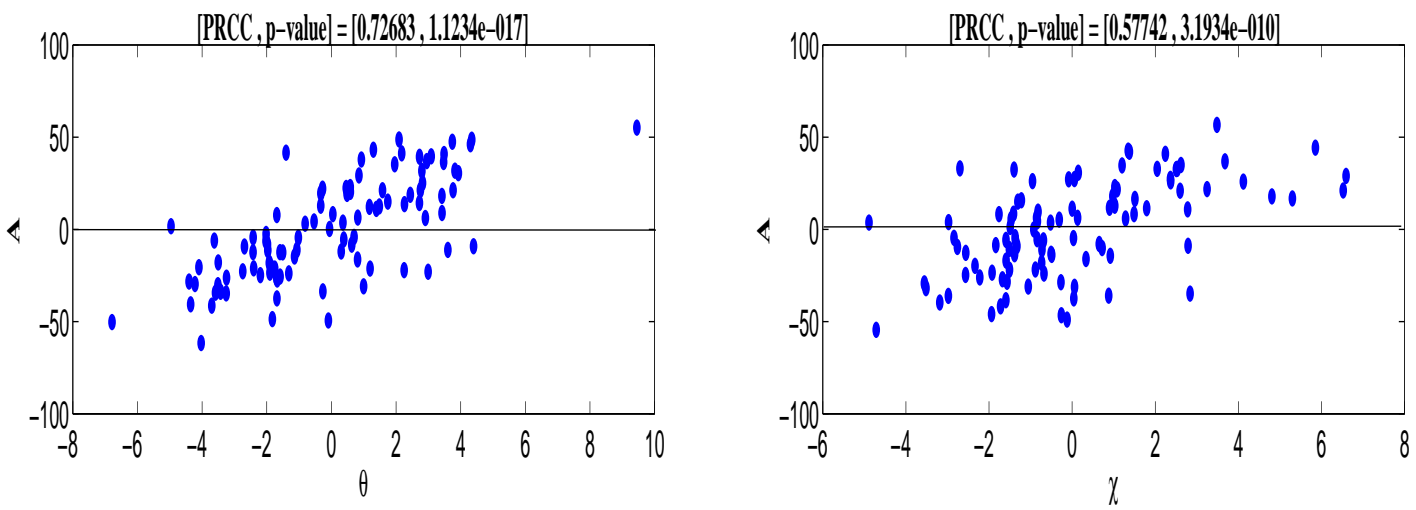

$(a)$
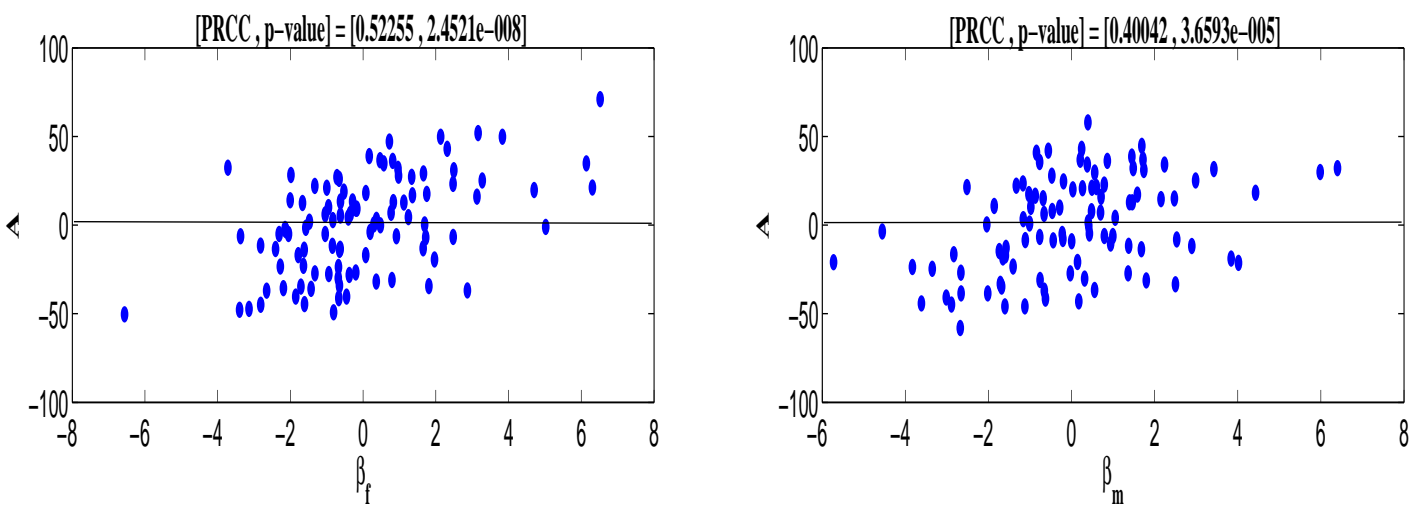

$(d)$

(c)
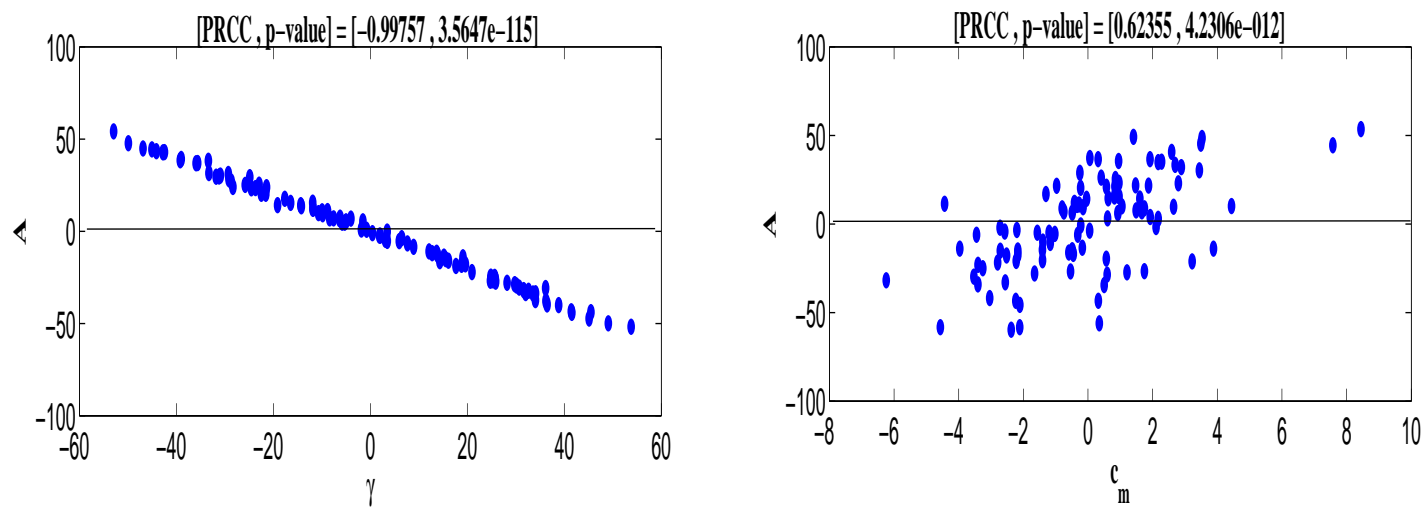

Figure 3: Partial rank correlation coefficients scatter plots of some of the parameters in $\mathcal{R}_{H}$ against the combined number of female and male AIDS cases. The baseline parameter values are given in Table 2 . 


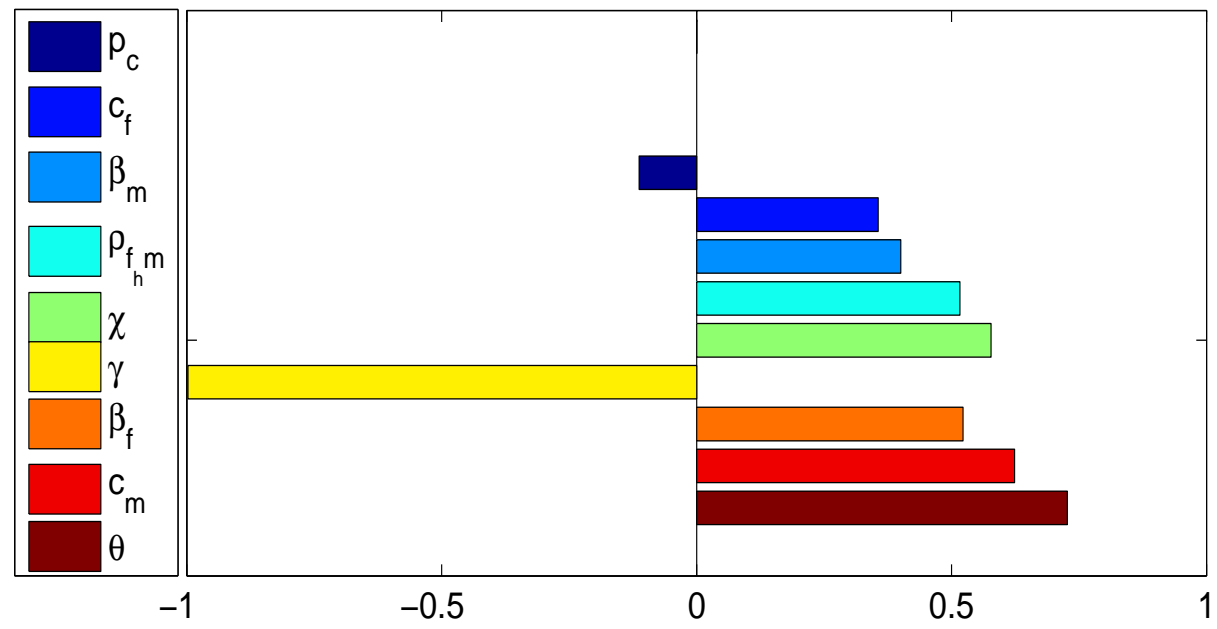

Figure 4: Partial rank correlation coefficients showing the effect of varying the input parameters on the response variable (combined female and male AIDS cases). All parameters with positive PRCCs will result in an increase on the number of AIDS cases when increased while an increase in parameters with negative PRCCs will result in reduced number of AIDS cases.

(a)

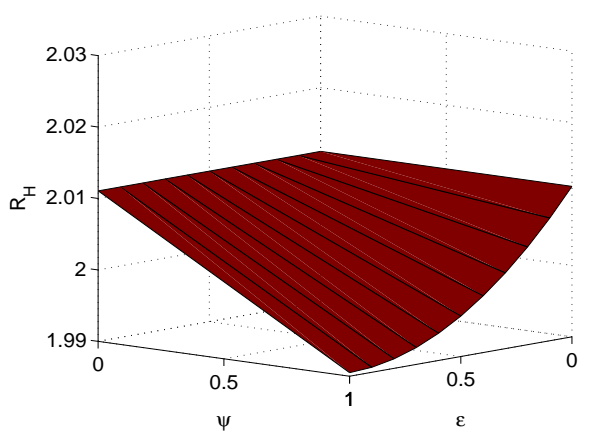

(c)

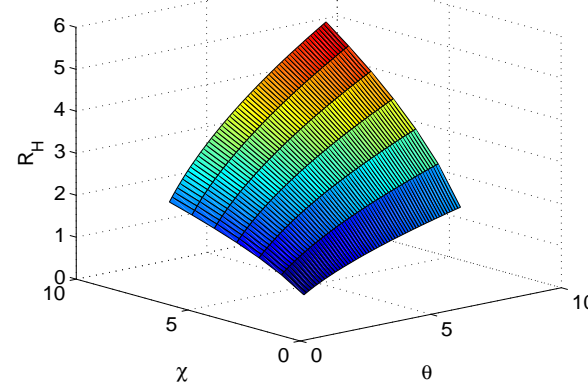

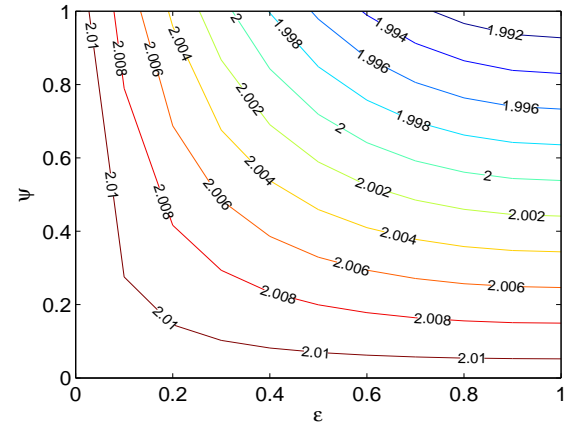

(b)

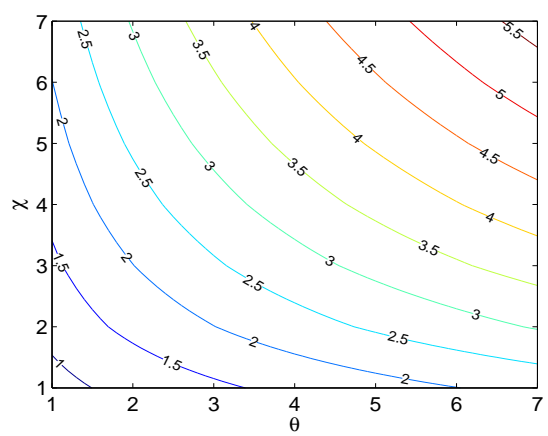

(d)

Figure 5: Plots: $(a)$ and $(b)$ show $\mathcal{R}_{H}$ against varying values of $\epsilon$ and $\psi,(c)$ and $(d)$ show $\mathcal{R}_{H}$ against varying values of $\theta$ and $\chi$ with other parameter values as given in Table 2 . 
(a)
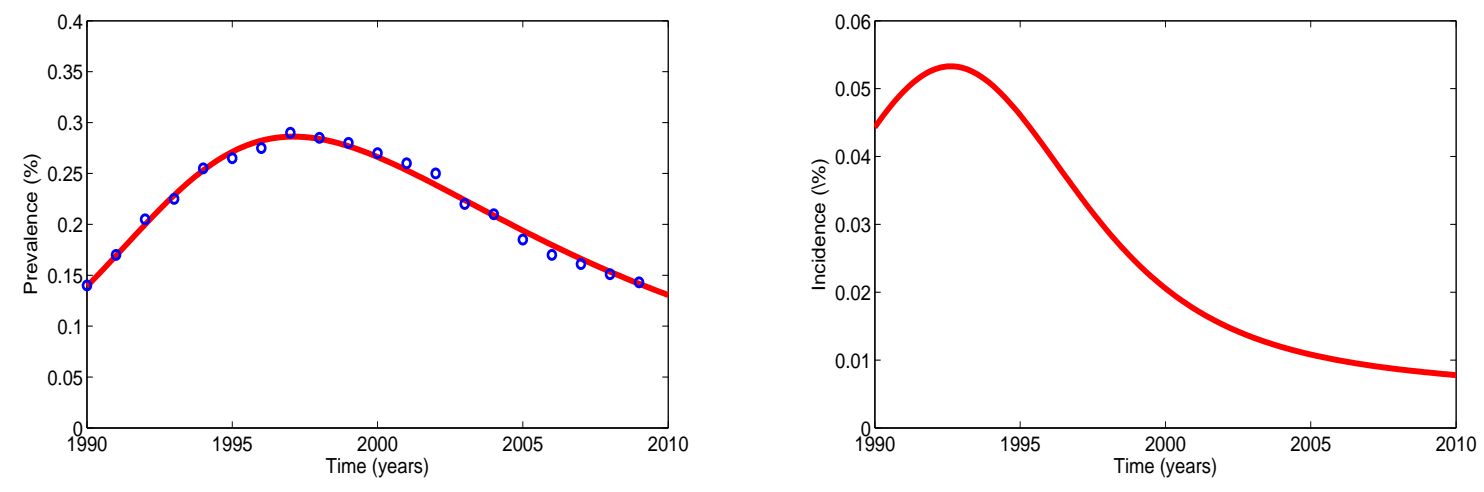

(c)
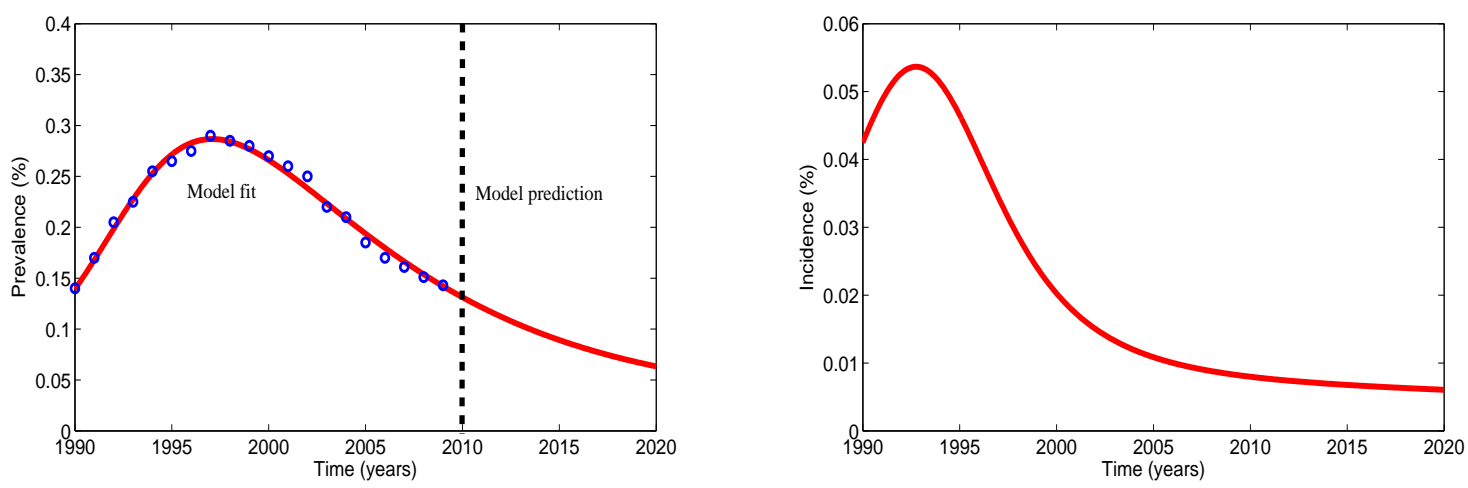

Figure 6: (a) Model fit to UNAIDS and MoHCW prevalence data for Zimbabwe. (b) Incidence curve corresponding to the prevalence from our model fit to UNAIDS and MoHCW data for Zimbabwe. (c) HIV model projection of prevalence till 2020. (d) HIV model projection of incidence till year 2020. UNAIDS and MoHCW data was used to fit the model and parameter values giving the best fit are given in Table 2. 
(a)
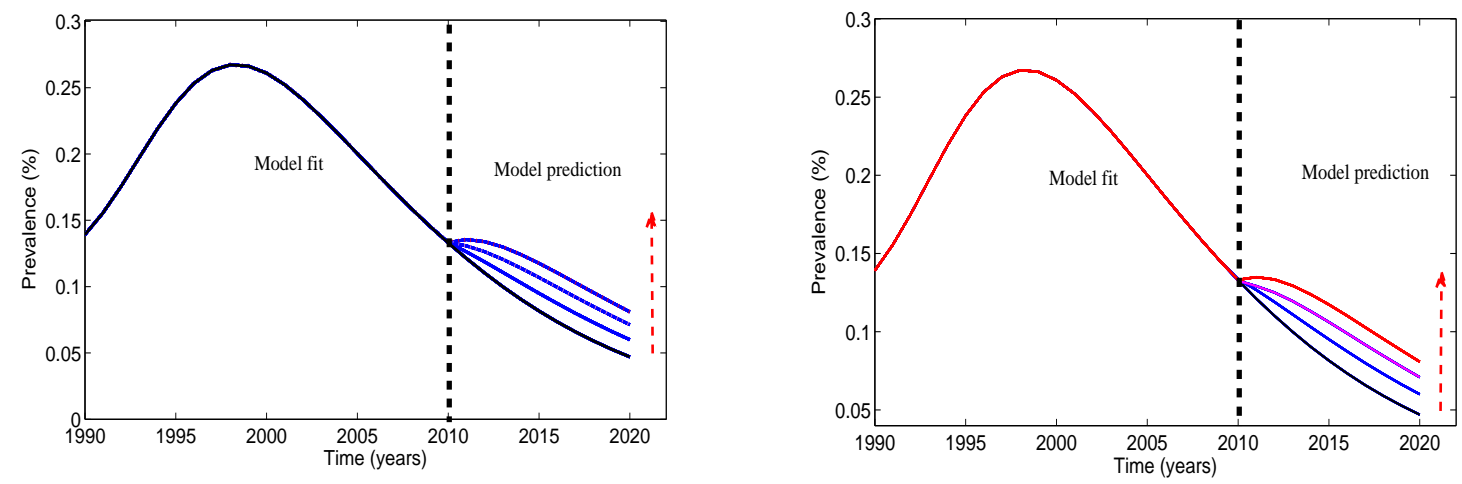

(b)

$(c)$
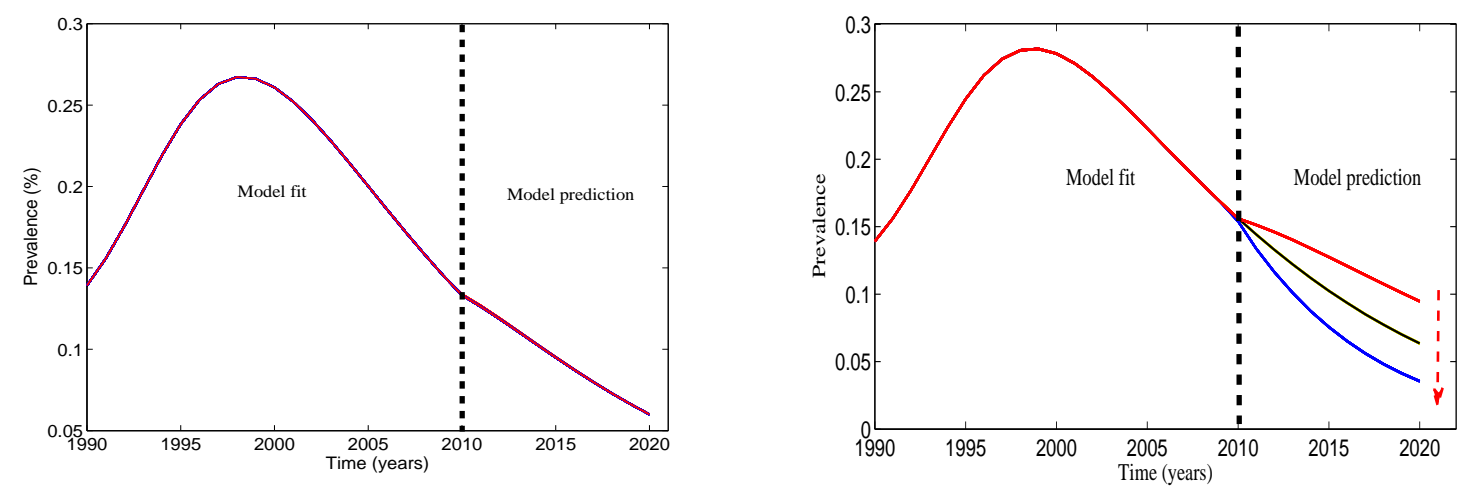

Figure 7: (a) Time series plot of HIV prevalence for increasing susceptibility and infectivity due to HC use from baseline case $(\theta, \chi)=1$ to 2,3 and 4 in the direction of the arrow. (b) Time series plot of HIV prevalence for increasing infectivity (with susceptibility unchanged) due to $\mathrm{HC} \theta$, from a baseline value of 1 to 4 in the direction of the arrow. (c) Time series plot of HIV prevalence for increasing suceptibility (with infectivity constant) due to $\mathrm{HC}$ use $\chi$, from baseline value of 1 to 4 in the direction of the arrow. Prevalence increases in the cohort using HC but remains unchanged in the general population since that population is very small. (d) Time series plot of HIV prevalence as the population using condoms as birth control tool increases from $0 \%, 30 \%$ to $100 \%$ in the direction of the arrow. Other parameter values are as given in Table 2 


\section{Tables}

\begin{tabular}{lcc|lcc}
\hline Parameter & PRCC & $p$-value & Parameter & PRCC & $p$-value \\
\hline$\gamma$ & -0.997 & $<0.001$ & $c_{f}$ & 0.356 & $<0.001$ \\
$\theta$ & 0.726 & $<0.001$ & $\rho_{f_{c} m}$ & 0.093 & 0.352 \\
$c_{m}$ & 0.623 & $<0.001$ & $\rho_{f_{n} m}$ & -0.079 & 0.431 \\
$\chi$ & 0.577 & $<0.001$ & $p_{c}$ & 0.061 & 0.540 \\
$\rho_{f_{h} m}$ & 0.516 & $<0.001$ & $\delta_{2}$ & 0.047 & 0.630 \\
$\beta_{f}$ & 0.522 & $<0.001$ & $\delta_{3}$ & -0.025 & 0.804 \\
$\beta_{m}$ & 0.400 & $<0.001$ & $\delta_{1}$ & -0.024 & 0.810 \\
\hline
\end{tabular}

Table 1: The PRCCs are between the input parameter and the output values (total AIDS cases). The $p$-value quantifies the probability that the result is due to chance rather than a true relationship between the input parameter and the output variable. 


\section{Biological, epidemiological and demographic parameters}

$\mu \quad$ Natural death rate

$\Lambda \quad$ Newly sexually matured individuals

$\phi \quad$ Proportion of new recruits who are female

$\alpha \quad$ Emigration rate

$\left(\beta_{f}, \beta_{m}\right) \quad$ Probability of HIV transmission

$\gamma \quad$ Average incubation period

$\left(c_{f}, c_{m}\right) \quad$ Frequency of partner acquisition

$\nu \quad$ AIDS induced death rate

$\varpi \quad$ Response parameter for behaviour change

\section{Birth control related parameters}

$\delta_{1} \quad$ Proportion of mature females who use condoms for birth control

Proportion of mature females who

use natural methods for birth control

Proportion of mature females who use

$\mathrm{HC}$ methods for birth control

$\rho_{m f_{c}} \quad$ Proportion of contacts made by males with females who use condoms for birth control

$\rho_{m f_{n}}$

Proportion of contacts made by males with

females who use natural methods for birth control

$\rho_{m f_{h}}$

Proportion of contacts made by males with

females who use $\mathrm{HC}$ methods for birth control

$\chi$

Relative measure of increased susceptibility

of females who use HC methods of birth control

Relative measure of increased infectivity

of females who use HC methods of birth control

Condom efficacy

Consistency of condom use

$\psi$

0.02
$100000 / \mathrm{yr}$
variable
0.01
$[0-1]$
0.125
$3[1-13]$ partners/yr
0.333
$>0$

$>0$

variable
variable
variable
variable
variable
variable
$\geq 2$
$\geq 2$
$85 \%[85-90] \%$
$0-25 \%$

$0-1$
$[58,59,61]$

$[59]$

$[58-61]$

$[15]$

$[58-61]$

$[5,74]$

$[58-61]$

0.055

$(0.51,0.8)$

0.095

$-$

$3.78 \times 10^{-6}$

Table 2: Model parameters and their interpretation. 